\title{
TOPOLOGY OF THE REPRESENTATION VARIETIES WITH BOREL MOLD FOR UNSTABLE CASES
}

\author{
KAZUNORI NAKAMOTO ${ }^{\bowtie}$ and TAKESHI TORII
}

(Received 12 December 2008; accepted 1 October 2010)

Communicated by J. Du

\begin{abstract}
In this paper we show that, in the stable case, when $m \geq 2 n-1$, the cohomology ring $H^{*}\left(\operatorname{Rep}_{n}(m)_{\mathrm{B}}\right)$ of the representation variety with Borel mold $\operatorname{Rep}_{n}(m)_{B}$ and $H^{*}\left(F_{n}\left(\mathbb{C}^{m}\right)\right) \otimes H^{*}\left(\operatorname{Flag}\left(\mathbb{C}^{n}\right)\right) \otimes \Lambda\left(s_{1}, \ldots, s_{n-1}\right)$ are isomorphic as algebras. Here the degree of $s_{i}$ is $2 m-3$ when $1 \leq i<n$. In the unstable cases, when $m \leq 2 n-2$, we also calculate the cohomology group $H^{*}\left(\operatorname{Rep}_{n}(m)_{\mathrm{B}}\right)$ when $n=3$, 4 . In the most exotic case, when $m=2, \operatorname{Rep}_{n}(2)_{B}$ is homotopy equivalent to $F_{n}\left(\mathbb{C}^{2}\right) \times \operatorname{PGL}_{n}(\mathbb{C})$, where $F_{n}\left(\mathbb{C}^{2}\right)$ is the configuration space of $n$ distinct points in $\mathbb{C}^{2}$. We regard $\operatorname{Rep}_{n}(2)_{B}$ as a scheme over $\mathbb{Z}$, and show that the Picard group $\operatorname{Pic}\left(\operatorname{Rep}_{n}(2)_{B}\right)$ of $\operatorname{Rep}_{n}(2)_{B}$ is isomorphic to $\mathbb{Z} / n \mathbb{Z}$. We give an explicit generator of the Picard group.
\end{abstract}

2010 Mathematics subject classification: primary 14D22; secondary 20M30, 20C99.

Keywords and phrases: moduli of representations, representation variety, Borel mold, free monoid.

\section{Introduction}

Moduli spaces of representations have been investigated and applied by many mathematicians in various subjects. For example, Fricke spaces were constructed as Teichmüller spaces of compact Riemann surfaces of genus $g$ (where $g \geq 2$ ) by using moduli spaces of discrete and faithful representations of the fundamental groups in $\mathrm{PSL}_{2}(\mathbb{R})$ or $\mathrm{SL}_{2}(\mathbb{R})$ [1]. The moduli spaces of stable vector bundles on a compact Riemann surface were described as the moduli spaces of irreducible unitary representations of the fundamental group [10,11]. The moduli spaces of $\theta$-semistable representations of quivers were constructed by King [5]. King's construction can be applied to developing the representation theory of wild algebras and to describing moduli spaces of vector bundles on special projective varieties.

In this paper, we continue our work from our paper [9] to investigate the topology of representation varieties with Borel mold more precisely. The objects with which we deal here are not irreducible representations, but representations with Borel mold.

(c) 2011 Australian Mathematical Publishing Association Inc. 1446-7887/2011 \$16.00 
By a global representation theory we mean a theory of representations parametrized by schemes or topological spaces. Recall that a mold is a subsheaf of $O_{X}$-subalgebras of the full matrix ring $\mathrm{M}_{n}\left(O_{X}\right)$ that is a subbundle of $\mathrm{M}_{n}\left(O_{X}\right)$ on ringed spaces $\left(X, O_{X}\right)$. Several moduli spaces of representations have been constructed for given types of molds. For example, in [7] we treat the moduli space of absolutely irreducible representations, and in [6] we treat the moduli space of representations with Borel mold. We propose to construct the moduli space with any mold of degree two for general groups and monoids in a future paper.

Recall that a representation with Borel mold for a group or a monoid is a representation that can be normalized to a representation in upper triangular matrices and whose image of the group or the monoid generates the algebra of upper triangular matrices. The moduli space of representations with Borel mold is important, since representations with Borel mold are typical examples of indecomposable representations. This moduli space is also easy to deal with in place of the absolutely irreducible case. In [9], we described the moduli space of representations with Borel mold for free monoids as fibre bundles over the configuration spaces. Our description has enabled us to determine the cohomology ring of the moduli space $\mathrm{Ch}_{n}(m)_{B}$ of representations with Borel mold of degree $n$ for the free monoid of rank $m$ over $\mathbb{C}$. We have also determined the cohomology rings of all of the related varieties $\mathbf{B}_{n}(m)_{B}$ and $\operatorname{Rep}_{n}(m)_{B}$ over $\mathbb{C}$ except for $\operatorname{Rep}_{n}(m)_{B}$ when $m \leq \frac{1}{2}\left(n^{2}-n\right)+1$.

In this article we deal with the variety $\operatorname{Rep}_{n}(m)_{B}$ where $m \leq \frac{1}{2}\left(n^{2}-n\right)+1$. The variety $\operatorname{Rep}_{n}(m)_{B}$ is defined to be the scheme consisting of representations with Borel mold of degree $n$ for the free monoid of rank $m$ (without taking the quotient by $\mathrm{PGL}_{n}$ ). The varieties $\operatorname{Rep}_{n}(m)_{B}$ behave differently when $m \geq 2 n-1$ and when $m \leq 2 n-2$. We call the case where $m \geq 2 n-1$ the stable case and the case where $m \leq 2 n-2$ the unstable case. In the stable case, we can easily describe the cohomology ring of $\operatorname{Rep}_{n}(m)_{B}$ over $\mathbb{C}$, as we see in the following theorem.

Theorem 1.1 (Corollary 3.3). If $m \geq 2 n-1$, then $H^{*}\left(\operatorname{Rep}_{n}(m)_{\mathrm{B}}\right)$ and

$$
H^{*}\left(F_{n}\left(\mathbb{C}^{m}\right)\right) \otimes H^{*}\left(\operatorname{Flag}\left(\mathbb{C}^{n}\right)\right) \otimes \Lambda\left(s_{1}, \ldots, s_{n-1}\right)
$$

are isomorphic as algebras. Here the degree of $s_{i}$ is $2 m-3$ when $1 \leq i<n$.

On the other hand, in the unstable case we cannot deal with the cohomology group of $\operatorname{Rep}_{n}(m)_{B}$ by a standard method. If $m<2 n-1$, then the free monoid of rank $m$ does not have enough free elements to generate the algebra of upper triangular matrices in $\mathrm{M}_{n}$ as algebras. Indeed, the $(2 n-1)$-matrices $\left\{E_{i i}\right\}_{1 \leq i \leq n}$ and $\left\{E_{i, i+1}\right\}_{1 \leq i<n}$ are 'natural' generators of the algebra of upper triangular matrices, while there are no 'natural' generators consisting of $(2 n-2)$-matrices. The smallest number of elements that 'naturally' generate the algebra of upper triangular matrices is $2 n-1$. In the unstable case, the cohomology of $\operatorname{Rep}_{n}(m)_{B}$ has a strange aspect, since the $m$-matrices are not free enough. This interpretation is not mathematical, but calculating the cohomology seems to indicate that this is the case. In Section 2, we calculate the cohomology group in the unstable case for small degree, namely, $n=3,4$. 
The case where $m=2$ is the most exotic of the unstable cases. The variety $\operatorname{Rep}_{n}(2)_{B}$ over $\mathbb{C}$ is homotopy equivalent to $F_{n}\left(\mathbb{C}^{2}\right) \times \mathrm{PGL}_{n}(\mathbb{C})$ (Theorem 5.2). In particular, by Corollary 5.3

$$
H^{2}\left(\operatorname{Rep}_{n}(2)_{B} ; \mathbb{Z}\right) \cong \mathbb{Z} / n \mathbb{Z}
$$

When we regard $\operatorname{Rep}_{n}(2)_{B}$ as a scheme over $\mathbb{Z}$, the Picard group $\operatorname{Pic}\left(\operatorname{Rep}_{n}(2)_{B}\right)$ is isomorphic to $\mathbb{Z} / n \mathbb{Z}$ (see Proposition 5.9). Further, we can describe the Picard group more precisely, as follows.

THeorem 1.2 (Theorem 5.12). For the universal flag

$$
\{0\} \subset \mathcal{L}_{1} \subset \mathcal{L}_{2} \subset \cdots \subset \mathcal{L}_{n}=O_{\operatorname{Rep}_{n}(2)_{B}}^{n}
$$

on the variety $\operatorname{Rep}_{n}(2)_{B}$ over $\mathbb{Z}$, we put $L_{i}:=\mathcal{L}_{i} / \mathcal{L}_{i-1}$ where $i=1,2, \ldots, n$. Then

$$
L_{1} \cong L_{2} \cong \cdots \cong L_{n}
$$

Furthermore, $L_{1}$ gives a generator of

$$
\operatorname{Pic}\left(\operatorname{Rep}_{n}(2)_{B}\right) \cong \mathbb{Z} / n \mathbb{Z}
$$

Here we use the term universal flag to mean the unique stable flag of $O_{\operatorname{Rep}_{n}(2)_{B}}^{\oplus n}$ under the action of the free monoid of rank $m$. As an application of this theorem we obtain the following result of global representation theory.

Corollary 1.3 (Corollary 5.15). Let $X$ be an affine scheme. Let $\rho$ be a representation with Borel mold of degree $n$ on $X$ for a group or a monoid $\Gamma$ generated by two elements. Suppose that Pic $X$ has no n-torsion elements. Then $\rho$ has the unique $\Gamma$-stable flag

$$
\{0\} \subset O_{X} \subset O_{X}^{2} \subset \cdots \subset O_{X}^{n} .
$$

In other words, there exists a suitable matrix $P \in \mathrm{GL}_{n}(R)$ such that $P^{-1} \rho(\gamma) P$ is an upper triangular matrix for each $\gamma \in \Gamma$ where $R$ is the coordinate ring of $X$.

We also discuss conditions under which there exists a two-dimensional representation with Borel mold for the free group of rank two over the rings of integers of quadratic fields. For the ring $R$ of integers of the quadratic field $\mathbb{Q}(\sqrt{m})$ such a twodimensional representation exists if and only if $m=-3$, or $m>0$ with $m \equiv 5 \bmod 8$ and there exists $\varepsilon=\frac{1}{2}(x+y \sqrt{m}) \in R^{\times}$for some odd integers $x, y$ (see Corollary 5.18).

In this paper we do not discuss several topics on the moduli of representations with Borel mold. The rational homotopy types of the moduli space $\mathrm{Ch}_{n}(m)_{B}$ and the related varieties $\mathbf{B}_{n}(m)_{B}$ and $\operatorname{Rep}_{n}(m)_{B}$ over $\mathbb{C}$ are discussed in [8], and we plan to discuss the characteristic classes of representations with Borel mold in future papers.

The organization of this paper is as follows. In Section 2 we review the representation variety with Borel mold and the results obtained in [9]. In Section 3 we deal with the stable case. In Section 4 we deal with the small degree cases. In Section 5 we discuss the case when $m=2$.

In this paper we use $H^{*}(X)$ to denote the cohomology group of $X$ with coefficients in $\mathbb{Z}$ for simplicity. 


\section{A survey of the representation variety with Borel mold}

This section is devoted to a survey of the representation variety with Borel mold $\operatorname{Rep}_{n}(m)_{B}$ of degree $n$ for the free monoid of rank $m$ and the related varieties $\mathrm{B}_{n}(m)_{B}$ and $\mathrm{Ch}_{n}(m)_{B}$. For more precise details see [9].

The variety $\operatorname{Rep}_{n}(m)_{B}$ is defined to be the subset of $\mathrm{M}_{n}(\mathbb{C})^{m}$ consisting of $m$-tuples $\left(A_{1}, \ldots, A_{m}\right)$ such that $A_{1}, \ldots, A_{m}$ generate a Borel mold:

$$
\operatorname{Rep}_{n}(m)_{B}:=\left\{\left(A_{1}, A_{2}, \ldots, A_{m}\right) \in \mathrm{M}_{n}(\mathbb{C})^{m} \mid A_{1}, \ldots, A_{m} \text { generate a Borel mold }\right\} .
$$

Here we say that a subalgebra of $\mathrm{M}_{n}(\mathbb{C})$ is a Borel mold if it can be written in the form $P \cdot \mathcal{B}_{n}(\mathbb{C}) \cdot P^{-1}$ for some $P \in \mathrm{GL}_{n}(\mathbb{C})$ where $\mathcal{B}_{n}(\mathbb{C})$ is the subalgebra of upper triangular matrices. We also define the variety $\mathrm{B}_{n}(m)_{B}$ by

$$
\mathrm{B}_{n}(m)_{B}:=\left\{\left(A_{1}, A_{2}, \ldots, A_{m}\right) \in \mathcal{B}_{n}(\mathbb{C})^{m} \mid A_{1}, \ldots, A_{m} \text { generate a Borel mold }\right\} .
$$

The group $\mathrm{PGL}_{n}(\mathbb{C})$ acts on $\operatorname{Rep}_{n}(m)_{B}$ by

$$
\left(A_{1}, \ldots, A_{m}\right) \mapsto\left(P^{-1} A_{1} P, \ldots, P^{-1} A_{m} P\right) .
$$

The quotient

$$
\mathrm{Ch}_{n}(m)_{B}:=\operatorname{Rep}_{n}(m)_{B} / \mathrm{PGL}_{n}(\mathbb{C})
$$

is called the moduli space of representations with Borel mold of degree $n$ for the free monoid of rank $m$.

Let Flag $\left(\mathbb{C}^{n}\right)$ be the flag variety, which consists of complete flags in $\mathbb{C}^{n}$. We define the morphism $\operatorname{Rep}_{n}(m)_{B} \rightarrow \operatorname{Flag}\left(\mathbb{C}^{n}\right)$ by letting $\left(A_{1}, \ldots, A_{m}\right)$ correspond to the unique flag that is invariant under $A_{1}, \ldots, A_{m}$. This morphism induces a fibration

$$
\mathrm{B}_{n}(m)_{B} \rightarrow \operatorname{Rep}_{n}(m)_{B} \rightarrow \operatorname{Flag}\left(\mathbb{C}^{n}\right) .
$$

Hence we obtain the associated Serre spectral sequence

$$
E_{2}^{p, q} \cong H^{p}\left(\operatorname{Flag}\left(\mathbb{C}^{n}\right)\right) \otimes H^{q}\left(\mathrm{~B}_{n}(m)_{B}\right) \Rightarrow H^{p+q}\left(\operatorname{Rep}_{n}(m)_{B}\right)
$$

We now recall the cohomology of $\mathrm{B}_{n}(m)_{B}$. For $\left(A_{1}, \ldots, A_{m}\right) \in \mathrm{B}_{n}(m)_{B}$ we denote by $a(i)_{j k}$ the $(j, k)$-entry of $A_{i}$. Put $w_{i}=\left(a(1)_{i i}, a(2)_{i i}, \ldots, a(m)_{i i}\right)$. We obtain the morphism $\mathrm{B}_{n}(m)_{B} \rightarrow F_{n}\left(\mathbb{C}^{m}\right)$ by

$$
\left(A_{1}, \ldots, A_{m}\right) \mapsto\left(w_{1}, w_{2}, \ldots, w_{n}\right)
$$

where

$$
F_{n}\left(\mathbb{C}^{m}\right):=\left\{\left(p_{1}, p_{2}, \ldots, p_{n}\right) \in\left(\mathbb{C}^{m}\right)^{n} \mid p_{1}, \ldots, p_{n} \text { are distinct }\right\}
$$

is the configuration space of $n$ distinct points in $\mathbb{C}^{m}$. This induces a fibration

$$
Y_{B} \rightarrow \mathrm{B}_{n}(m)_{B} \rightarrow F_{n}\left(\mathbb{C}^{m}\right)
$$

with $Y_{B} \simeq\left(S^{2 m-3}\right)^{n-1}$. The associated Serre spectral sequence collapses from $E_{2}$ term. This leads to the following theorem. 
Theorem 2.1 (See [9, Theorem 4.3]). The cohomology ring of $\mathrm{B}_{n}(m)_{B}$ is an exterior algebra generated by $s_{1}, \ldots, s_{n-1}$ over $H^{*}\left(F_{n}\left(\mathbb{C}^{m}\right)\right)$. That is,

$$
H^{*}\left(\mathrm{~B}_{n}(m)_{B}\right) \cong H^{*}\left(F_{n}\left(\mathbb{C}^{m}\right)\right) \otimes \Lambda\left(s_{1}, \ldots, s_{n-1}\right) .
$$

Here deg $s_{i}=2 m-3$.

The cohomology of $\mathrm{Ch}_{n}(m)_{B}$ is also easy to describe. There is an isomorphism between $\mathrm{Ch}_{n}(m)_{B}$ and $\mathrm{B}_{n}(m)_{B} / \mathrm{B}_{n}(\mathbb{C})$ where $\mathrm{B}_{n}(\mathbb{C})$ is the subgroup of $\mathrm{PGL}_{n}(\mathbb{C})$ consisting of upper triangular matrices. Then we obtain a fibration

$$
Y_{C} \rightarrow \mathrm{Ch}_{n}(m)_{B} \rightarrow F_{n}\left(\mathbb{C}^{m}\right)
$$

where $Y_{C} \simeq\left(\mathbb{C} \mathbb{P}^{m-2}\right)^{n-1}$. The associated Serre spectral sequence collapses from the $E_{2}$ term.

THEOREM 2.2 (See [9, Theorem 5.2]). The cohomology ring of $\mathrm{Ch}_{n}(m)_{B}$ is a truncated polynomial algebra generated by $t_{1}, \ldots, t_{n-1}$ over $H^{*}\left(F_{n}\left(\mathbb{C}^{m}\right)\right)$. That is,

$$
H^{*}\left(\mathrm{Ch}_{n}(m)_{B}\right) \cong H^{*}\left(F_{n}\left(\mathbb{C}^{m}\right)\right) \otimes \mathbb{Z}\left[t_{1}, \ldots, t_{n-1}\right] /\left(t_{1}^{m-1}, \ldots, t_{n-1}^{m-1}\right),
$$

where $\operatorname{deg} t_{j}=2$.

Let us return to the cohomology of $\operatorname{Rep}_{n}(m)_{B}$. In the spectral sequence

$$
E_{2}^{p, q} \cong H^{p}\left(\operatorname{Flag}\left(\mathbb{C}^{n}\right)\right) \otimes H^{q}\left(\mathrm{~B}_{n}(m)_{B}\right) \Rightarrow H^{p+q}\left(\operatorname{Rep}_{n}(m)_{B}\right),
$$

the image of $H^{*}\left(F_{n}\left(\mathbb{C}^{m}\right)\right)$ in $H^{*}\left(\mathrm{~B}_{n}(m)_{B}\right)$ consists of permanent cycles [9, Lemma 6.3]. If $m>\frac{1}{2}\left(n^{2}-n\right)+1$, then we obtain the following proposition.

Proposition 2.3 (See [9, Proposition 6.5]). If $m>\frac{1}{2}\left(n^{2}-n\right)+1$, then the spectral sequence collapses from the $E_{2}$ term. In this case,

$$
H^{*}\left(\operatorname{Rep}_{n}(m)_{B}\right) \cong H^{*}\left(F_{n}\left(\mathbb{C}^{m}\right)\right) \otimes H^{*}\left(\operatorname{Flag}\left(\mathbb{C}^{n}\right)\right) \otimes \Lambda\left(s_{1}, \ldots, s_{n-1}\right)
$$

where the degree of $s_{i}$ is $2 m-3$ when $i=1, \ldots, n-1$.

In the general case, the spectral sequence does not always collapse. In order to describe the $E_{2 m-1}$ term, we prepare the following. For positive numbers $n$ and $m$ we define the differential graded algebra $C_{n}(m)$ by

$$
C_{n}(m):=\mathbb{Z}\left[t_{1}, t_{2}, \ldots, t_{n}\right] /\left(c_{1}, c_{2}, \ldots, c_{n}\right) \otimes \Lambda\left(s_{1}, s_{2}, \ldots, s_{n-1}\right)
$$

where $c_{i}$ is the $i$ th elementary symmetric polynomial in $\mathbb{Z}\left[t_{1}, t_{2}, \ldots, t_{n}\right]$, deg $t_{i}=2$, $\operatorname{deg} s_{i}=2 m-3$ and $d\left(s_{j}\right)=\left(t_{j}-t_{j+1}\right)^{m-1}$ when $j=1,2, \ldots, n-1$.

Lemma 2.4 (See [9, Lemma 6.6]). The $E_{2 m-1}$ term of the Serre spectral sequence of the fibre bundle

$$
\mathrm{B}_{n}(m)_{B} \rightarrow \operatorname{Rep}_{n}(m)_{B} \rightarrow \operatorname{Flag}\left(\mathbb{C}^{n}\right)
$$

is $H^{*}\left(C_{n}(m)\right) \otimes H^{*}\left(F_{n}\left(\mathbb{C}^{m}\right)\right)$. 
Before ending this section, we introduce the case when $n=m=2$.

Proposition 2.5 (See [9, Proposition 6.7]). If $n=2$ and $m=2$, then there is a homotopy equivalence

$$
\operatorname{Rep}_{2}(2)_{B} \simeq F_{2}\left(\mathbb{C}^{2}\right) \times \mathrm{PU}(2) \simeq S^{3} \times \mathbb{R} \mathbb{P}^{3}
$$

Hence the cohomology ring of $\operatorname{Rep}_{2}(2)_{B}$ is given by

$$
H^{*}\left(\operatorname{Rep}_{2}(2)_{B}\right) \cong H^{*}\left(S^{3}\right) \otimes H^{*}\left(\mathbb{R P}^{3}\right) .
$$

In particular, $H^{*}\left(\operatorname{Rep}_{2}(2)_{\mathrm{B}}\right)$ has the following module structure.

$$
\begin{array}{c|cccccccc}
i & 0 & 1 & 2 & 3 & 4 & 5 & 6 & \geq 7 \\
\hline H^{i}\left(\operatorname{Rep}_{2}(2)_{\mathrm{B}}\right) & \mathbb{Z} & \{0\} & \mathbb{Z} / 2 \mathbb{Z} & \mathbb{Z}^{2} & \{0\} & \mathbb{Z} / 2 \mathbb{Z} & \mathbb{Z} & \{0\}
\end{array}
$$

\section{The stable case}

In this section we show that if $m \geq 2 n-1$, then the spectral sequence

$$
E_{2}^{p, q} \cong H^{p}\left(\operatorname{Flag}\left(\mathbb{C}^{n}\right)\right) \otimes H^{q}\left(\mathrm{~B}_{n}(m)_{B}\right) \Rightarrow H^{p+q}\left(\operatorname{Rep}_{n}(m)_{B}\right)
$$

collapses. We say that the case where $m \geq 2 n-1$ is stable and that the other case is unstable. Indeed, the differentials in the spectral sequence vanish if and only if the case is stable.

Recall that the cohomology ring of the flag variety Flag $\left(\mathbb{C}^{n}\right)$ is given by

$$
H^{*}\left(\operatorname{Flag}\left(\mathbb{C}^{n}\right)\right)=\mathbb{Z}\left[t_{1}, t_{2}, \ldots, t_{n}\right] /\left(c_{1}, c_{2}, \ldots, c_{n}\right)
$$

where $c_{i}$ is the $i$ th elementary symmetric polynomial in $\mathbb{Z}\left[t_{1}, t_{2}, \ldots, t_{n}\right]$. Note that we can take

$$
\left\{t_{1}^{m_{1}} t_{2}^{m_{2}} \cdots t_{n}^{m_{n}} \mid 0 \leq m_{i} \leq n-i\right\}
$$

as a basis of

$$
H^{*}\left(\operatorname{Flag}\left(\mathbb{C}^{n}\right)\right)=\mathbb{Z}\left[t_{1}, t_{2}, \ldots, t_{n}\right] /\left(c_{1}, c_{2}, \ldots, c_{n}\right) .
$$

For details see [4, Section 14.2]. Let $I$ be the ideal generated by $c_{1}, \ldots, c_{n}$.

Lemma 3.1. When $1 \leq i \leq n$ and the $i$ indices $j_{1}, j_{2}, \ldots, j_{i} \in\{1,2, \ldots, n\}$ are distinct,

$$
t_{j_{1}}^{n-1} t_{j_{2}}^{n-2} t_{j_{3}}^{n-3} \cdots t_{j_{k}}^{n-k} \cdots t_{j_{i-1}}^{n-i+1} t_{j_{i}}^{n-i} \cdot t_{j_{i}} \in I
$$

Proof. It suffices to prove that

$$
t_{1}^{n-1} t_{2}^{n-2} \cdots t_{k}^{n-k} \cdots t_{i-1}^{n-i+1} t_{i}^{n-i} \cdot t_{i} \in I
$$


because $I$ is invariant under the action of the symmetric group $S_{n}$ on the set of indices $\{1,2, \ldots, n\}$. We prove this statement by induction on $i$. First, we prove the statement in the case when $i=1$. Considering $t_{1}^{n} \bmod I$,

$$
\begin{aligned}
t_{1}^{n}=t_{1}^{n-1} \cdot t_{1} & \equiv t_{1}^{n-1}\left(-t_{2}-t_{3}-\cdots-t_{n}\right) \\
& \equiv-t_{1}^{n-2}\left(\sum_{1<i} t_{1} t_{i}\right) \\
& \equiv-t_{1}^{n-2}\left(-\sum_{1<i<j} t_{i} t_{j}\right) \\
& \equiv t_{1}^{n-3}\left(\sum_{1<i<j} t_{1} t_{i} t_{j}\right) \\
& \equiv \cdots \\
& \equiv(-1)^{n-1} t_{1} t_{2} t_{3} \cdots t_{n} \equiv 0 .
\end{aligned}
$$

This implies that the statement is true in the case when $i=1$.

Now let $2 \leq m \leq n$ and suppose that the statement is true for all values of $i$ less than or equal to $m-1$. Note that

$$
t_{1}^{n-1} \equiv(-1)^{n-1} \sum_{1<i_{1}<i_{2}<\cdots i_{n-1}} t_{i_{1}} t_{i_{2}} \cdots t_{i_{n-1}} \equiv(-1)^{n-1} t_{2} t_{3} \cdots t_{n} .
$$

In the case when $i=m$, we see that

$$
\begin{aligned}
& t_{1}^{n-1} t_{2}^{n-2} \cdots t_{k}^{n-k} \cdots t_{i-1}^{n-i+1} t_{i}^{n-i} \cdot t_{i} \\
& \equiv(-1)^{n-1}\left(t_{2} t_{3} \cdots t_{n}\right) \cdot t_{2}^{n-2} \cdots t_{k}^{n-k} \cdots t_{i-1}^{n-i+1} t_{i}^{n-i} \cdot t_{i} \\
& \equiv(-1)^{n-1} t_{2}^{n-1} t_{3}^{n-2} \cdots t_{k}^{n-k+1} \cdots t_{i-1}^{n-i+2} t_{i}^{n-i+1} \cdot t_{i} \cdot\left(t_{i+1} \cdots t_{n}\right) \\
& \equiv 0 .
\end{aligned}
$$

Our result follows by induction.

Proposition 3.2. If $m \geq 2 n-1$, then all differentials of $C_{n}(m)$ are 0 . In particular, $H\left(C_{n}(m)\right)=C_{n}(m)$.

Proof. Note that $d\left(s_{i}\right)=\left(t_{i}-t_{i+1}\right)^{m-1}$ is expressed as a linear combination $t_{i}^{\alpha} t_{i+1}^{\beta}$ with $\alpha+\beta=m-1$. If $m \geq 2 n-1$, then $\left(t_{i}-t_{i+1}\right)^{m-1}=0$ by Lemma 3.1. This implies that all differentials of $C_{n}(m)$ are 0 .

Corollary 3.3. If $m \geq 2 n-1$, then the cohomology ring of $\operatorname{Rep}_{n}(m)_{\mathrm{B}}$ is given by

$$
H^{*}\left(\operatorname{Rep}_{n}(m)_{B}\right) \cong H^{*}\left(F_{n}\left(\mathbb{C}^{m}\right)\right) \otimes H^{*}\left(\operatorname{Flag}\left(\mathbb{C}^{n}\right)\right) \otimes \Lambda\left(s_{1}, \ldots, s_{n-1}\right),
$$

where the degree of $s_{i}$ is $2 m-3$ when $1 \leq i<n$.

Proof. The statement follows from Proposition 3.2. 
By Corollary 3.3 we only need to consider the unstable cases in what follows. The following table shows the bound of the unstable range $m \leq 2 n-2$.

\begin{tabular}{c|ccccccccccccccc}
$n$ & 2 & 3 & 4 & 5 & 6 & 7 & 8 & 9 & 10 & 11 & 12 & 13 & 14 & 15 & $\cdots$ \\
\hline $2 n-2$ & 2 & 4 & 6 & 8 & 10 & 12 & 14 & 16 & 18 & 20 & 22 & 24 & 26 & 28 & $\cdots$
\end{tabular}

We show that the differentials never vanish in $C_{n}(m)$ if $m \leq 2 n-2$.

Proposition 3.4. If $m \leq 2 n-2$, then $d\left(s_{i}\right) \neq 0$ in $C_{n}(m)$ for all $1 \leq i<n$.

Proof. By symmetry, it suffices to show that $d\left(s_{1}\right) \neq 0$. Indeed, we may assume that $m=2 n-2$ for if the relation $d\left(s_{1}\right)=\left(t_{1}-t_{2}\right)^{2 n-3} \neq 0$ holds for the case when $m=2 n-2$, then the relation $d\left(s_{1}\right)=\left(t_{1}-t_{2}\right)^{m-1} \neq 0$ also holds if $m<2 n-2$. Since $t_{1}^{n} \equiv t_{2}^{n} \equiv 0 \bmod I$

$$
d\left(s_{1}\right)=\left(t_{1}-t_{2}\right)^{2 n-3} \equiv(-1)^{n} c\left(t_{1}^{n-1} t_{2}^{n-2}-t_{1}^{n-2} t_{2}^{n-1}\right),
$$

where $c=\left(\begin{array}{c}2 n-3 \\ n-1\end{array}\right)$.

Let $N$ be the top degree (that is, $n(n-1))$ component of $\mathbb{Z}\left[t_{1}, \ldots, t_{n}\right] / I$. Then it is easy to check that $N$ is a free module of rank one over $\mathbb{Z}$ generated by the class $t_{1}^{n-1} t_{2}^{n-2} \cdots t_{n-1}$ and the action of the symmetric group $S_{n}$ on $N$ is the sign representation. Let $a=t_{3}^{n-3} t_{4}^{n-4} \cdots t_{n-1}$. Then

$$
d\left(s_{1}\right) a=(-1)^{n} 2 c t_{1}^{n-1} t_{2}^{n-2} a \neq 0 .
$$

This completes the proof.

\section{Small degree cases}

In unstable cases, we need to calculate the cohomology group of $\operatorname{Rep}_{n}(m)_{B}$ directly for each case. This section is devoted to calculating the cohomology group of $\operatorname{Rep}_{n}(m)_{B}$ and its Poincaré series in small degree cases. The unstable case when $(n, m)=(2,2)$ has been discussed in Proposition 2.5 .

First we consider the cases when $n=3$. When $n=3$, there are three unstable cases, namely, $m=2,3,4$. We next consider the cases when $n=4$ and $2 \leq m \leq 6$.

Recall that the cohomology group of $F_{n}\left(\mathbb{C}^{m}\right)$ is free over any commutative ring $R$ and its Poincaré series is given by

$$
\operatorname{PS}\left(F_{n}\left(\mathbb{C}^{m}\right)\right):=\sum_{i \geq 0} \operatorname{rank}_{R} H^{*}\left(F_{n}\left(\mathbb{C}^{m}\right) ; R\right) \cdot t^{i}=\prod_{\alpha=1}^{n-1}\left(1+\alpha t^{2 m-1}\right) .
$$

4.1. The space $Y_{\boldsymbol{R}}$. Recall that there is a fibre bundle $Y_{B} \rightarrow \mathrm{B}_{n}(m)_{B} \rightarrow F_{n}\left(\mathbb{C}^{m}\right)$ and $\operatorname{Rep}_{n}(m)_{B}$ is isomorphic to $\mathrm{B}_{n}(m)_{B} \times_{\mathrm{B}_{n}} \mathrm{PGL}_{n}$. We set $Y_{R}=Y_{B} \times_{\mathrm{B}_{n}} \mathrm{PGL}_{n}$. Then there is a fibre bundle $Y_{B} \rightarrow Y_{R} \rightarrow \operatorname{Flag}\left(\mathbb{C}^{n}\right)$. 
In the case when $m=2$ we can determine the homotopy type of the space $Y_{R}$.

LeMma 4.1. When $m=2$, we have $Y_{R} \simeq \operatorname{PGL}_{n}(\mathbb{C})$.

Proof. Recall that $\mathrm{B}_{n}$ acts freely on $Y_{B}$ and the quotient is $Y_{C}$. Thus we have a fibre bundle $\mathrm{PGL}_{n} \rightarrow Y_{R} \rightarrow Y_{C}$. Since $Y_{C}$ is homotopy equivalent to $\left(\mathbb{C P}^{m-2}\right)^{n-1}$ we have that $Y_{C}$ is contractible if $m=2$. Hence the map $\mathrm{PGL}_{n} \rightarrow Y_{R}$ is a homotopy equivalence.

In [8] we have shown that $\operatorname{Rep}_{n}(m)_{B}$ is rationally homotopy equivalent to the product $F_{n}\left(\mathbb{C}^{m}\right) \times Y_{R}$. If $p$ is sufficiently large, then the similar result on the $p$-local homotopy type of $\operatorname{Rep}_{n}(m)_{B}$ holds.

Lemma 4.2. Let $p$ be a prime number such that $p \geq m$. Then $\operatorname{Rep}_{n}(m)_{B}$ is p-locally homotopy equivalent to $F_{n}\left(\mathbb{C}^{m}\right) \times Y_{R}$.

Proof. In [9, Lemma 4.4], we defined a subspace $\mathrm{B}_{n}(m)_{B}^{\prime}$ of $\mathrm{B}_{n}(m)_{B}$ such that the inclusion $\mathrm{B}_{n}(m)_{B}^{\prime} \hookrightarrow \mathrm{B}_{n}(m)_{B}$ is a $\mathrm{T}_{\mathbb{R}}$-homotopy equivalence. Let $Y_{B}^{\prime}$ be the fibre of the fibre bundle $\mathrm{B}_{n}(m)_{B}^{\prime} \rightarrow F_{n}\left(\mathbb{C}^{m}\right)$ that is homotopy equivalent to the product of $(n-1)$ copies of $S^{2 m-3}$. Set

$$
\operatorname{Rep}_{n}(m)_{B}^{\prime}=\mathrm{B}_{n}(m)_{B}^{\prime} \times_{\mathrm{T}_{\mathbb{R}}} \mathrm{PU}(n)
$$

and

$$
Y_{R}^{\prime}=Y_{B}^{\prime} \times_{\mathrm{T}_{\mathbb{R}}} \mathrm{PU}(n)
$$

Then there is a fibre bundle

$$
Y_{R}^{\prime} \rightarrow \operatorname{Rep}_{n}(m)_{B}^{\prime} \rightarrow F_{n}\left(\mathbb{C}^{m}\right) .
$$

The canonical maps $\operatorname{Rep}_{n}(m)_{B}^{\prime} \rightarrow \operatorname{Rep}_{n}(m)_{B}$ and $Y_{R}^{\prime} \rightarrow Y_{R}$ are homotopy equivalences.

When $i \neq j$, we let

$$
\pi_{i, j}: F_{n}\left(\mathbb{C}^{m}\right) \rightarrow F_{2}\left(\mathbb{C}^{m}\right)
$$

be the map

$$
\pi_{i, j}\left(x_{1}, \ldots, x_{n}\right)=\left(x_{i}, x_{j}\right) .
$$

There is a homotopy equivalence $F_{2}\left(\mathbb{C}^{m}\right) \stackrel{\simeq}{\longrightarrow} S^{2 m-1}$, given by $(x, y) \mapsto\|x-y\|^{-1}$ $(x-y)$.

Let $E$ be a contractible free $\mathrm{U}(m-1)$-space and let $X=E / \mathrm{U}(m-2)$. There is a fibre bundle $S^{2 m-3} \rightarrow X \rightarrow B \mathrm{U}(m-1)$, where $B \mathrm{U}(m-1)=E / \mathrm{U}(m-1)$. The fibre bundle

$$
S^{2 m-3} \rightarrow \mathrm{U}(m) / \mathrm{U}(m-2) \rightarrow \mathrm{U}(m) / \mathrm{U}(m-1)=S^{2 m-1}
$$

is the induced bundle of $X \rightarrow B \mathrm{U}(m-1)$ by the map $S^{2 m-1} \rightarrow B \mathrm{U}(m-1)$ that is a generator of

$$
\pi_{2 m-1}(B \mathrm{U}(m-1)) \cong \mathbb{Z} /(m-1) ! \mathbb{Z}
$$

When $1 \leq i<n$, we let $X_{i}$ be a copy of $X$. There is a fibre bundle

$$
Y_{R}^{\prime} \rightarrow\left(\prod_{i} X_{i}\right) \times_{\mathrm{T}_{\mathbb{R}}} \mathrm{PU}(n) \rightarrow \prod_{i} B \mathrm{U}(m-1) .
$$


The fibre bundle $\operatorname{Rep}_{n}(m)_{B}^{\prime} \rightarrow F_{n}\left(\mathbb{C}^{m}\right)$ is the induced bundle by the map

$$
F_{n}\left(\mathbb{C}^{m}\right) \stackrel{\prod_{i} \pi_{i, i+1}}{\longrightarrow} \prod_{i} F_{2}\left(\mathbb{C}^{m}\right) \simeq \prod_{i} S^{2 m-1} \rightarrow \prod_{i} B \mathrm{U}(m-1) .
$$

If $p \geq m$, then $\pi_{2 m-1}(B \mathrm{U}(m-1))_{(p)}=\{0\}$. Hence the fibration

$$
\operatorname{Rep}_{n}(m)_{B(p)}^{\prime} \rightarrow F_{n}\left(\mathbb{C}^{m}\right)_{(p)}
$$

is induced by the trivial map to $\prod B \mathrm{U}(m-1)_{(p)}$. Therefore

$$
\operatorname{Rep}_{n}(m)_{B(p)}^{\prime} \simeq F_{n}\left(\mathbb{C}^{m}\right)_{(p)} \times Y_{R(p)}^{\prime} .
$$

This completes the proof.

Corollary 4.3. Let $p$ be a prime number such that $p \geq m$ and let $R$ be a commutative $\mathbb{Z}_{(p)}$-algebra. Then there is an isomorphism of graded algebras:

$$
H^{*}\left(\operatorname{Rep}_{n}(m)_{B} ; R\right) \cong H^{*}\left(F_{n}\left(\mathbb{C}^{m}\right) ; R\right) \otimes_{R} H^{*}\left(Y_{R} ; R\right) .
$$

Let $R$ be a commutative ring. We denote by $E_{r}^{*, *}(C)$ the Serre spectral sequence associated to the fibre bundle $Y_{R} \rightarrow \operatorname{Rep}_{n}(m)_{B} \rightarrow F_{n}\left(\mathbb{C}^{m}\right)$ with coefficients in $R$. That is,

$$
E_{2}^{*, *}(C) \cong H^{*}\left(F_{n}\left(\mathbb{C}^{m}\right) ; R\right) \otimes_{R} H^{*}\left(Y_{R} ; R\right) \Longrightarrow H^{*}\left(\operatorname{Rep}_{n}(m)_{B} ; R\right)
$$

Proposition 4.4. Suppose that $H^{*}\left(\operatorname{Rep}_{n}(m)_{B} ; R\right) \rightarrow H^{*}\left(Y_{R} ; R\right)$ is a split surjection of $R$-modules (or R-algebras). Then there is an isomorphism of $H^{*}\left(F_{n}\left(\mathbb{C}^{m}\right) ; R\right)$-modules (or $R$-algebras respectively), namely

$$
H^{*}\left(\operatorname{Rep}_{n}(m)_{B} ; R\right) \cong H^{*}\left(F_{n}\left(\mathbb{C}^{m}\right) ; R\right) \otimes_{R} H^{*}\left(Y_{R} ; R\right) .
$$

Proof. By the assumption, $E_{r}^{*, *}(C)$ collapses from the $E_{2}$ term. The splitting map $H^{*}\left(Y_{R}\right) \rightarrow H^{*}\left(\operatorname{Rep}_{n}(m)_{B}\right)$ extends to a homomorphism of $H^{*}\left(F_{n}\left(\mathbb{C}^{m}\right)\right)$-modules

$$
H^{*}\left(F_{n}\left(\mathbb{C}^{m}\right)\right) \otimes H^{*}\left(Y_{R}\right) \rightarrow H^{*}\left(\operatorname{Rep}_{n}(m)_{B}\right)
$$

This map is an isomorphism since the map of associated graded modules is an isomorphism.

Recall that the differential graded algebra $C_{n}(m)$ is defined to be

$$
C_{n}(m)=\mathbb{Z}\left[t_{1}, \ldots, t_{n}\right] /\left(c_{1}, \ldots, c_{n}\right) \otimes \Lambda\left(s_{1}, \ldots, s_{n-1}\right)
$$

with $d\left(t_{i}\right)=0$ and $d\left(s_{i}\right)=\left(t_{i}-t_{i+1}\right)^{m-1}$. We give a bigrading on $C_{n}(m)$ by $\left|t_{i}\right|=(2,0)$ and $\left|s_{i}\right|=(0,2 m-3)$ and let $C_{n}(m)^{p, q}$ denote the degree $(p, q)$-component of $C_{n}(m)$.

Let

$$
\omega=t_{1}^{n-1} t_{2}^{n-2} \cdots t_{n-1} \otimes s_{1} \cdots s_{n-1} \in C_{n}(m) .
$$


When $i+p=n(n-1)$ and $j+q=(2 m-3)(n-1)$, we define a pairing

$$
\langle-,-\rangle: C_{n}(m)^{i, j} \otimes C_{n}(m)^{p, q} \rightarrow \mathbb{Z}
$$

by $\langle x, y\rangle=\alpha$ if $x y=\alpha \omega$. Since $C_{n}(m)$ is isomorphic to the cohomology ring of the orientable compact manifold Flag $\left(\mathbb{C}^{n}\right) \times\left(S^{2 m-3}\right)^{n-1}$, it is a Poincaré duality algebra. Hence the pairing $\langle-,-\rangle$ is perfect; that is, the pairing induces an isomorphism

$$
C_{n}(m)^{i, j} \stackrel{\cong}{\longrightarrow}\left(C_{n}^{p, q}(m)\right)^{\vee},
$$

where $\left(C^{p, q}\right)^{\vee}=\operatorname{Hom}_{\mathbb{Z}}\left(C^{p, q}, \mathbb{Z}\right)$. We denote by $d^{\vee}: C_{n}(m)^{\vee} \rightarrow C_{n}(m)^{\vee}$ the dual of $d: C_{n}(m) \rightarrow C_{n}(m)$.

Lemma 4.5. The following diagram commutes up to sign,

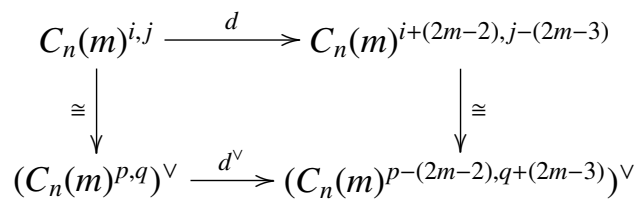

where $i+p=n(n-1)$ and $j+q=(2 m-3)(n-1)$.

Proof. It is sufficient to show that $d(x) \cdot y=(-1)^{i+j+1} x \cdot d(y)$ whenever $|x|=(i, j)$ and $|y|=(p-(2 m-2), q+(2 m-3))$. For reasons of degree, $x \cdot y=0$. Thus we have

$$
0=d(x \cdot y)=d(x) \cdot y+(-1)^{i+j} x \cdot d(y) .
$$

This implies that $d(x) \cdot y=(-1)^{i+j+1} x \cdot d(y)$, as required.

Let $E_{r}^{*, *}(Y)$ be the Serre spectral sequence associated to the fibre bundle

$$
Y_{B} \rightarrow Y_{R} \rightarrow \operatorname{Flag}\left(\mathbb{C}^{n}\right)
$$

with coefficients in a commutative ring $R$ :

$$
E_{2}^{*, *}(Y)=H^{*}\left(\operatorname{Flag}\left(\mathbb{C}^{n}\right) ; R\right) \otimes_{R} H^{*}\left(Y_{B} ; R\right) \Longrightarrow H^{*}\left(Y_{R} ; R\right)
$$

The first possible nontrivial differential is $d_{2 m-2}$ and there is an isomorphism of differential graded algebras

$$
\left(E_{2 m-2}(Y), d_{2 m-2}\right) \cong\left(C_{n}(m ; R), d_{R}\right),
$$

where $C_{n}(m ; R)=C_{n}(m) \otimes R$ and $d_{R}=d \otimes 1_{R}$.

We let $E_{r}^{* * *}(P)$ denote the Serre spectral sequence associated to the fibre bundle

$$
\mathrm{B}_{n}(m)_{B} \rightarrow \operatorname{Rep}_{n}(m)_{B} \rightarrow \operatorname{Flag}\left(\mathbb{C}^{n}\right)
$$


with coefficients in a commutative ring $R$ :

$$
E_{2}^{*, *}(P) \cong H^{*}\left(\operatorname{Flag}\left(\mathbb{C}^{n}\right) ; R\right) \otimes_{R} H^{*}\left(\mathrm{~B}_{n}(m)_{B} ; R\right) \Longrightarrow H^{*}\left(\operatorname{Rep}_{n}(m)_{B} ; R\right) .
$$

There is a map of fibre bundles,

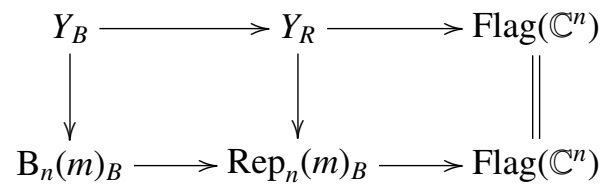

which induces a map of spectral sequences

$$
E_{r}^{* * *}(P) \rightarrow E_{r}^{*, *}(Y)
$$

4.2. The cases when $\boldsymbol{n}=3$. In this subsection we consider the unstable cases where $(n, m)$ is one of $(3,2),(3,3)$ and $(3,4)$. We study the cohomology of $\operatorname{Rep}_{3}(m)_{B}$ when $m=2,3,4$, and its Poincaré series.

If $m=2$, then the following proposition follows from Theorem 5.2 below.

Proposition 4.6. In the case when $(n, m)=(3,2)$, there is an isomorphism of R-algebras:

$$
H^{*}\left(\operatorname{Rep}_{3}(2)_{B} ; R\right) \cong H^{*}\left(F_{3}\left(\mathbb{C}^{2}\right) ; R\right) \otimes_{R} H^{*}\left(\mathrm{PGL}_{3}(\mathbb{C}) ; R\right)
$$

for any commutative ring $R$. The Poincaré series of $\operatorname{Rep}_{3}(2)_{B}$ is given by

$$
\operatorname{PS}\left(\operatorname{Rep}_{3}(2)_{B} ; k\right)=\operatorname{PS}\left(F_{3}\left(\mathbb{C}^{2}\right)\right) \cdot \operatorname{PS}\left(\operatorname{PGL}_{3}(\mathbb{C}) ; k\right)
$$

for any field $k$.

Remark 4.7. In this case, $Y_{R} \simeq \mathrm{PGL}_{3}(\mathbb{C})$ by Lemma 4.1 .

If $p$ is a prime number other than 3 , then $\mathrm{PGL}_{3}(\mathbb{C})$ is $p$-locally homotopy equivalent to $\mathrm{SU}(3)$. The modulo 3 cohomology ring of $\mathrm{PGL}_{3}(\mathbb{C})$ is

$$
H^{*}\left(\mathrm{PGL}_{3}(\mathbb{C}) ; \mathbb{Z} / 3 \mathbb{Z}\right)=(\mathbb{Z} / 3 \mathbb{Z})[y] /\left(y^{3}\right) \otimes \Lambda\left(e_{1}, e_{3}\right)
$$

where $|y|=2$ and $\left|e_{i}\right|=i$. Hence the Poincaré series of $Y_{R} \simeq \operatorname{PGL}_{3}(\mathbb{C})$ is given as follows.

$$
\operatorname{PS}\left(Y_{R} ; \mathbb{Z} / p \mathbb{Z}\right)= \begin{cases}\left(1+t^{3}\right)\left(1+t^{5}\right) & \text { if } p \neq 3, \\ (1+t)\left(1+t^{2}+t^{4}\right)\left(1+t^{3}\right) & \text { if } p=3 .\end{cases}
$$

Next we study the cohomology group of $Y_{R}$ in the case when $(n, m)=(3,3)$. In a

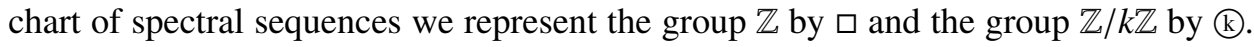
We also abbreviate $\mathbb{Z}^{\oplus r}$ and $(\mathbb{Z} / k \mathbb{Z})^{\oplus r}$ to $\square^{r}$ and $\gtrless^{r}$, respectively. 
Lemma 4.8. When $(n, m)=(3,3)$, the spectral sequence $E_{r}^{*, *}(Y)$ collapses from the $E_{5}$ term for any $R$. If $R=\mathbb{Z}$, then the $E_{\infty}$ term $H^{*, *}\left(C_{3}(3)\right)=\bigoplus_{p, q} H\left(C_{3}(3)\right)^{p, q}$ is given as follows.

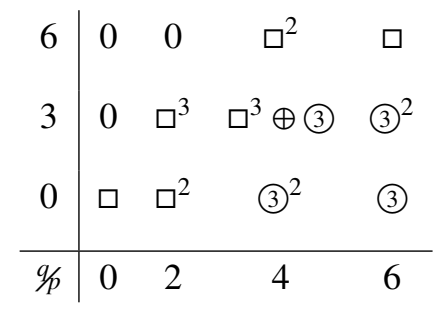

The additive generators are

$$
\begin{array}{cl}
1, t_{1}, t_{2}, t_{1}^{2}, t_{1} t_{2}, t_{1}^{2} t_{2}, & (q=0), \\
a, b, c, a t_{1}, b t_{1}, b t_{2}, c t_{1}, b t_{1}^{2}, c t_{1}^{2}, & (q=3), \\
a b, a c, a b t_{1} & (q=6),
\end{array}
$$

where $\left|t_{1}\right|=\left|t_{2}\right|=(2,0),|a|=|b|=|c|=(2,3)$. The relations are

$$
\begin{gathered}
t_{2}^{2}=-t_{1}^{2}-t_{1} t_{2}, \quad t_{1}^{3}=0, \quad 3 t_{1}^{2}=3 t_{1} t_{2}=0, \\
a^{2}=0, \quad b^{2}=0, \quad c^{2}=0, \\
b c=-a c, \quad a t_{2}=-b t_{2}+c t_{1}, \quad c t_{2}=-a t_{1}-b t_{1}-c t_{1}, \\
3 c t_{1}=0, \quad a t_{1}^{2}=0, \quad b t_{1} t_{2}=0, \\
a b t_{2}=0, \quad a c t_{1}=0 .
\end{gathered}
$$

The cohomology ring $H^{*}\left(Y_{R} ; \mathbb{Z}\right)$ and $H^{*}\left(C_{3}(3)\right)$ are isomorphic as graded commutative algebras.

Proof. For reasons of degree, the spectral sequence collapses from the $E_{5}$ term for any $R$. Hence $E_{\infty}=E_{5}=H\left(C_{3}(3 ; R)\right)$.

We shall compute $H\left(C_{3}(3)\right)$ where $R=\mathbb{Z}$. We write $C=C_{3}(3)$ and $H=H\left(C_{3}(3)\right)$, and take the following basis of $C^{*, 0}: 1, t_{1}, t_{2}, t_{1}^{2}, t_{1} t_{2}, t_{1}^{2} t_{2}$.

For reasons of degree, $H^{p, q}=C^{p, q}$ when $(p, q)=(0,0),(2,0),(4,6),(6,6)$. With respect to the above basis, $d\left(s_{1}\right)=-3 t_{1} t_{2}$ and $d\left(s_{2}\right)=-3 t_{1}^{2}$. Hence $H^{4,0}=\mathbb{Z} /(3)\left\{t_{1}^{2}, t_{1} t_{2}\right\}$ and $H^{0,3}=\{0\}$. If we set $a=s_{2} t_{1}, b=s_{1} t_{1}+s_{1} t_{2}$ and $c=s_{2} t_{2}-s_{1} t_{1}$, then one checks that $a, b$ and $c$ are cycles. We take a basis of $C^{2,3}$ to be $s_{1} t_{1}, a, b, c$. Then $d\left(s_{1} t_{1}\right)=$ $-3 t_{1}^{2} t_{2}$ and hence $H^{2,3}=\mathbb{Z}\{a, b, c\}$ and $H^{6,0}=\mathbb{Z} /(3)\left\{t_{1}^{2} t_{2}\right\}$.

We can now take a basis of $C^{4,3}$ to be $a t_{1}, b t_{1}, b t_{2}, c t_{1}$. Since

$$
d\left(s_{1} s_{2}\right)=3 s_{1} t_{1}^{2}-3 s_{2} t_{1} t_{2}=-3 c t_{1}
$$

we obtain

$$
H^{4,3}=\mathbb{Z}\left\{a t_{1}, b t_{1}, b t_{2}\right\} \oplus \mathbb{Z} /(3)\left\{c t_{1}\right\}
$$

and $H^{0,6}=\{0\}$. 
Since $b t_{1}^{2}=s_{1} t_{1}^{2} t_{2}$ and $c t_{1}^{2}=s_{2} t_{1}^{2} t_{2}$ we can take a basis of $C^{6,3}$ to be $b t_{1}^{2}, c t_{1}^{2}$. Since $d\left(s_{1} s_{2} t_{1}\right)=-3 c t_{1}^{2}$ and $d\left(s_{1} s_{2} t_{2}\right)=3 b t_{1}^{2}+3 c t_{1}^{2}$ we obtain $H^{6,3}=\mathbb{Z} /(3)\left\{b t_{1}^{2}, c t_{1}^{2}\right\}$ and $H^{2,6}=\{0\}$. A tedious, but straightforward, computation verifies the relations. By sparseness, for every $n$ there is only one nontrivial $H^{p, q}$ whose total degree is given by $p+q=n$. Hence $H^{*}\left(Y_{R} ; \mathbb{Z}\right) \cong H$ as graded modules. Furthermore, we see that there are no multiplicative extensions. Hence $H^{*}\left(Y_{R} ; \mathbb{Z}\right) \cong H$ as graded commutative algebras. This completes the proof.

Corollary 4.9. When $(n, m)=(3,3)$, the Poincaré series of $Y_{R}$ is given as follows:

$$
\begin{aligned}
& \operatorname{PS}\left(Y_{R} ; \mathbb{Z} / p \mathbb{Z}\right)=1+2 t^{2}+3 t^{5}+3 t^{7}+2 t^{10}+t^{12} \quad \text { if } p \neq 3, \\
& \operatorname{PS}\left(Y_{R} ; \mathbb{Z} / 3 \mathbb{Z}\right)=\left(1+t^{2}\right)\left(1+t^{2}+t^{4}\right)\left(1+t^{3}\right)^{2} .
\end{aligned}
$$

In the case when $(n, m)=(3,4)$, we have the following lemma.

Lemma 4.10. When $(n, m)=(3,4)$, the spectral sequence $E_{r}^{*, *}(Y)$ collapses from the $E_{7}$ term for any $R$. If $R=\mathbb{Z}$, the $E_{\infty}$ term $H^{*, *}\left(C_{3}(4)\right)=\bigoplus_{p, q} H\left(C_{3}(4)\right)^{p, q}$ is given as follows.

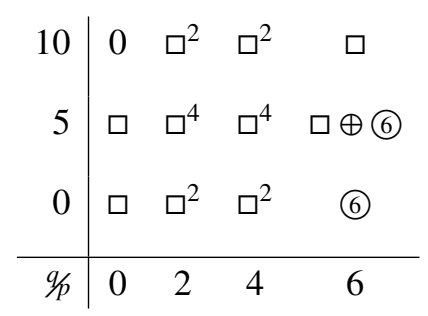

The additive generators are

$$
\begin{array}{cl}
1, t_{1}, t_{2}, t_{1}^{2}, t_{1} t_{2}, t_{1}^{2} t_{2}, & (q=0), \\
a, a t_{1}, a t_{2}, b, c, a t_{1}^{2}, a t_{1} t_{2}, b t_{1}, b t_{2}, b t_{1} t_{2}, a t_{1}^{2} t_{2} & (q=5), \\
a b, a c, a b t_{1}, a b t_{2}, a b t_{1} t_{2} & (q=10),
\end{array}
$$

where $\left|t_{1}\right|=\left|t_{2}\right|=(2,0),|a|=(0,5)$ and $|b|=|c|=(2,5)$. The relations are

$$
\begin{gathered}
t_{1}^{3}=0, \quad t_{2}^{2}=-t_{1}^{2}-t_{1} t_{2}, \quad 6 t_{1}^{2} t_{2}=0, \\
a^{2}=b^{2}=c^{2}=b c=0, \\
c t_{1}=b t_{2}, \quad c t_{2}=-b t_{1}-b t_{2}, \\
b t_{1}^{2}=0 .
\end{gathered}
$$

The cohomology ring $H^{*}\left(Y_{R} ; \mathbb{Z}\right)$ is isomorphic to $H^{*}\left(C_{3}(4)\right)$ as graded commutative algebras.

Proof. For reasons of degree, $E_{r}^{*, *}(Y)$ collapses from the $E_{7}$ term for any $R$. Hence $E_{\infty}=E_{7}=H\left(C_{3}(4 ; R)\right)$.

We shall compute $H\left(C_{3}(4)\right)$ where $R=\mathbb{Z}$. We write $C=C_{3}(4)$ and $H=H\left(C_{3}(4)\right)$, and take the basis of $C^{*, 0}$ given by $1, t_{1}, t_{2}, t_{1}^{2}, t_{1} t_{2}, t_{1}^{2} t_{2}$. 
For reasons of degree, $H^{p, q}=C^{p, q}$ unless $(p, q)=(6,0),(0,5),(6,5),(0,10)$. With respect to the basis above, $d\left(s_{1}\right)=d\left(s_{2}\right)=-6 t_{1}^{2} t_{2}$ and so $a=s_{1}-s_{2}$ is a cycle. We take a basis of $C^{0,5}$ given by $s_{1}, a$. Then $H^{6,0}=\mathbb{Z} /(6)\left\{t_{1}^{2} t_{2}\right\}$ and $H^{0,5}=\mathbb{Z}\{a\}$. We set $b=s_{2} t_{1}$ and $c=s_{2} t_{2}$. We can take the basis of $C^{6,5}$ given by $b t_{1} t_{2}, a t_{1}^{2} t_{2}$. Since $d\left(s_{1} s_{2}\right)=6 a t_{1}^{2} t_{2}$,

$$
H^{6,5}=\mathbb{Z}\left\{b t_{1} t_{2}\right\} \oplus \mathbb{Z} / 6\left\{a t_{1}^{2} t_{2}\right\},
$$

and $H^{0,10}=\{0\}$. A tedious, but straightforward, computation verifies the relations.

By sparseness, for every $n$ there is only one nontrivial $H^{p, q}$ whose total degree is given by $p+q=n$. Hence $H^{*}\left(Y_{R} ; \mathbb{Z}\right) \cong H$ as graded modules. Furthermore, we see that there are no multiplicative extensions. Hence $H^{*}\left(Y_{R} ; \mathbb{Z}\right) \cong H$ as graded commutative algebras. This completes the proof.

Corollary 4.11. When $(n, m)=(3,4)$, the Poincaré series of $Y_{R}$ is given as follows:

$$
\begin{aligned}
& \operatorname{PS}\left(Y_{R} ; \mathbb{Z} / p \mathbb{Z}\right)=\left(1+2 t^{2}+2 t^{4}+2 t^{7}+2 t^{9}+t^{11}\right)\left(1+t^{5}\right) \quad \text { if } p \neq 2,3, \\
& \operatorname{PS}\left(Y_{R} ; \mathbb{Z} / p \mathbb{Z}\right)=\left(1+t^{2}\right)\left(1+t^{2}+t^{4}\right)\left(1+t^{5}\right)^{2} \quad \text { if } p=2,3 .
\end{aligned}
$$

Theorem 4.12. When $n=3$ and $m \geq 2$, there is an isomorphism of $H^{*}\left(F_{3}\left(\mathbb{C}^{m}\right) ; R\right)$ modules:

$$
H^{*}\left(\operatorname{Rep}_{3}(m)_{B} ; R\right) \cong H^{*}\left(F_{3}\left(\mathbb{C}^{m}\right) ; R\right) \otimes_{R} H^{*}\left(Y_{R} ; R\right)
$$

for any commutative ring $R$. If $R=\mathbb{Z}$, then this is an isomorphism of algebras. The Poincaré series of $\operatorname{Rep}_{3}(m)_{B}$ is given by

$$
\operatorname{PS}\left(\operatorname{Rep}_{3}(m)_{B} ; k\right)=\operatorname{PS}\left(F_{3}\left(\mathbb{C}^{m}\right)\right) \cdot \operatorname{PS}\left(Y_{R} ; k\right)
$$

for any field $k$.

Proof. We may assume that $m \geq 3$ by Lemma 4.1 and Proposition 4.6. We have the map of spectral sequences $E_{r}^{*, *}(P) \rightarrow E_{r}^{*, *}(Y)$. By comparing the $E_{\infty}$ terms, we see that $H^{*}\left(\operatorname{Rep}_{n}(m)_{B} ; R\right) \rightarrow H^{*}\left(Y_{R} ; R\right)$ is a split surjection of $R$-modules. If $R=\mathbb{Z}$, then the map $H^{*}\left(\operatorname{Rep}_{n}(m)_{B} ; R\right) \rightarrow H^{*}\left(Y_{R} ; R\right)$ is also a split surjection of $\mathbb{Z}$-algebras. The theorem now follows by Proposition 4.4.

4.3. The cases when $\boldsymbol{n}=4$. In this subsection we deal with the unstable cases where $n=4$ and $2 \leq m \leq 6$. We study the cohomology of $\operatorname{Rep}_{4}(m)_{B}$ and its Poincaré series.

If $m=2$, then the following proposition follows from Theorem 5.2 below.

Proposition 4.13. When $(n, m)=(4,2)$, there is an isomorphism of R-algebras:

$$
H^{*}\left(\operatorname{Rep}_{4}(2)_{B} ; R\right) \cong H^{*}\left(F_{4}\left(\mathbb{C}^{2}\right) ; R\right) \otimes_{R} H^{*}\left(\mathrm{PGL}_{4}(\mathbb{C}) ; R\right)
$$

for any commutative ring $R$. The Poincaré series of $\operatorname{Rep}_{4}(2)_{B}$ is given by

$$
\operatorname{PS}\left(\operatorname{Rep}_{4}(2)_{B} ; k\right)=\operatorname{PS}\left(F_{4}\left(\mathbb{C}^{2}\right)\right) \cdot \operatorname{PS}\left(\mathrm{PGL}_{4}(\mathbb{C}) ; k\right)
$$

for any field $k$. 
Remark 4.14. In this case, $Y_{R} \simeq \mathrm{PGL}_{4}(\mathbb{C})$ by Lemma 4.1 .

If $p$ is an odd prime, then $\mathrm{PGL}_{4}(\mathbb{C})$ is $p$-locally homotopy equivalent to $\mathrm{SU}(4)$. The modulo 2 cohomology ring of $\mathrm{PGL}_{4}(\mathbb{C})$ is given by

$$
H^{*}\left(\mathrm{PGL}_{4}(\mathbb{C}) ; \mathbb{Z} / 2 \mathbb{Z}\right) \cong(\mathbb{Z} / 2 \mathbb{Z})[y] /\left(y^{4}\right) \otimes \Lambda\left(e_{1}, e_{3}, e_{5}\right),
$$

where $|y|=2$ and $\left|e_{i}\right|=i$. Hence the Poincaré series of $Y_{R} \simeq \mathrm{PGL}_{4}(\mathbb{C})$ is given as follows:

$$
\begin{aligned}
& \operatorname{PS}\left(Y_{R} ; \mathbb{Z} / p \mathbb{Z}\right)=\left(1+t^{3}\right)\left(1+t^{5}\right)\left(1+t^{7}\right) \quad \text { if } p \neq 2, \\
& \operatorname{PS}\left(Y_{R} ; \mathbb{Z} / 2 \mathbb{Z}\right)=\frac{1-t^{8}}{1-t^{2}}(1+t)\left(1+t^{3}\right)\left(1+t^{5}\right) .
\end{aligned}
$$

We next consider the case when $(n, m)=(4,3)$. Recall that in a chart of the spectral sequence we represent the group $\mathbb{Z}$ by $\square$ and the group $\mathbb{Z} / k \mathbb{Z}$ by $\ltimes$. We represent $\mathbb{Z}^{\oplus r}$ and $(\mathbb{Z} / k \mathbb{Z})^{\oplus r}$ by $\square^{r}$ and $\AA^{r}$, respectively. We can take a basis of $\mathbb{Z}\left[t_{1}, t_{2}, t_{3}, t_{4}\right] /\left(c_{1}, c_{2}, c_{3}, c_{4}\right)$ to be $\left\{t_{1}^{m_{1}} t_{2}^{m_{2}} t_{3}^{m_{3}} \mid 0 \leq m_{i} \leq 4-i\right\}$.

Proposition 4.15. When $(n, m)=(4,3)$, the spectral sequence $E_{r}^{*, *}(Y)$ collapses from the $E_{5}$ term for any $R$. If $R=\mathbb{Z}$, then the $E_{\infty}$ term $H^{* * *}\left(C_{4}(3)\right)$ is given as follows.

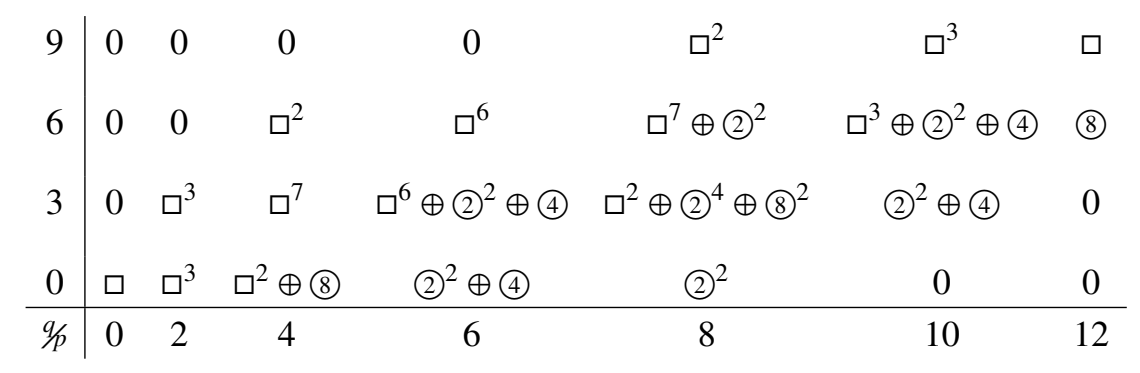

The cohomology group $H^{*}\left(Y_{R}\right)$ is isomorphic to $H^{*}\left(C_{4}(3)\right)$ as a graded module.

Proof. We write $C=C_{4}(4)$ and $H=H\left(C_{4}(4)\right)$, and set

$$
a=t_{1}^{2}-2 t_{1} t_{2}+t_{2}^{2}, \quad b=-t_{1}^{2}-t_{1} t_{2}-t_{1} t_{3}-3 t_{2} t_{3}, \quad c=-3 t_{1}^{2}-2 t_{1} t_{2}-3 t_{2}^{2} .
$$

Then $d\left(s_{1}\right)=a, d\left(s_{2}\right)=b$ and $d\left(s_{3}\right)=c$. Now we see easily that $C^{0,3}=\mathbb{Z}\left\{s_{1}, s_{2}, s_{3}\right\}$ and $C^{4,0}=\mathbb{Z}\left\{t_{1}^{2}, t_{1} t_{2}, t_{1} t_{3}, t_{2}^{2}, t_{2} t_{3}\right\}$, and we can verify that $d$ is injective and the cokernel of $d: C^{0,3} \rightarrow C^{4,0}$ is isomorphic to $\mathbb{Z}^{\oplus 2} \oplus \mathbb{Z} /(8)$ by using elementary transformations of matrices. Hence $H^{0,3}=\{0\}$ and $H^{4,0} \cong \mathbb{Z}^{\oplus 2} \oplus \mathbb{Z} /(8)$.

We have

$$
C^{2,3}=\mathbb{Z}\left\{s_{1}, s_{2}, s_{3}\right\} \otimes \mathbb{Z}\left\{t_{1}, t_{2}, t_{2}\right\}
$$

and

$$
C^{6,0}=\mathbb{Z}\left\{t_{1}^{3}, t_{1}^{2} t_{2}, t_{1}^{2} t_{3}, t_{1} t_{2}^{2}, t_{1} t_{2} t_{3}, t_{2}^{2} t_{3}\right\}
$$


The differential $d: C^{2,3} \rightarrow C^{6,0}$ is given by $d\left(s_{1} t_{i}\right)=a t_{i}, d\left(s_{2} t_{i}\right)=b t_{i}$ and $d\left(s_{3} t_{i}\right)=c t_{i}$ when $i=1,2,3$. We calculate that

$$
\begin{aligned}
& a t_{1}=t_{1}^{3}-2 t_{1}^{2} t_{2}+t_{1} t_{2}^{2}, \quad a t_{2}=-t_{1}^{3}-3 t_{1} t_{2}^{2}, \quad a t_{3}=t_{1}^{2} t_{3}-2 t_{1} t_{2} t_{3}+t_{2}^{2} t_{3}, \\
& b t_{1}=-t_{1}^{3}-t_{1}^{2} t_{2}-t_{1}^{2} t_{3}-3 t_{1} t_{2} t_{3}, \quad b t_{2}=-t_{1}^{2} t_{2}-t_{1} t_{2}^{2}-t_{1} t_{2} t_{3}-3 t_{2}^{2} t_{3}, \\
& b t_{3}=-2 t_{1}^{3}+t_{1}^{2} t_{2}+t_{1} t_{2}^{2}+3 t_{1} t_{2} t_{3}+3 t_{2}^{2} t_{3}, \\
& c t_{1}=-3 t_{1}^{3}-2 t_{1}^{2} t_{2}-3 t_{1} t_{2}^{2}, \quad c t_{2}=3 t_{1}^{3}+t_{1} t_{2}^{2}, \quad c t_{3}=-3 t_{1}^{2} t_{3}-2 t_{1} t_{2} t_{3}-3 t_{2}^{2} t_{3} .
\end{aligned}
$$

We can see that the kernel of $d$ is free and of rank 3, and the cokernel of $d$ is isomorphic to $\mathbb{Z} /(2)^{\oplus 2} \oplus \mathbb{Z} /(4)$ by using elementary transformations of matrices. Hence $H^{2,3} \cong \mathbb{Z}^{\oplus 3}$ and $H^{6,0} \cong \mathbb{Z} /(2)^{\oplus 2} \oplus \mathbb{Z} /(4)$.

We also have $C^{0,6}=\mathbb{Z}\left\{s_{1} s_{2}, s_{1} s_{3}, s_{2} s_{3}\right\}$ and

$$
C^{4,3}=\mathbb{Z}\left\{s_{1}, s_{2}, s_{3}\right\} \otimes \mathbb{Z}\left\{t_{1}^{2}, t_{1} t_{2}, t_{1} t_{3}, t_{2}^{2}, t_{2} t_{3}\right\} .
$$

The differential $d: C^{0,6} \rightarrow C^{4,3}$ is given by $d\left(s_{1} s_{2}\right)=a s_{2}-b s_{1}, d\left(s_{1} s_{3}\right)=a s_{3}-c s_{1}$ and $d\left(s_{2} s_{3}\right)=b s_{3}-c s_{2}$. It is easy to see that $d$ is injective and the image of $d$ is a direct summand of $C^{4,3}$. Hence $H^{0,6}=\{0\}$.

We have

$$
C^{8,0}=\mathbb{Z}\left\{t_{1}^{3} t_{2}, t_{1}^{3} t_{3}, t_{1}^{2} t_{2}^{2}, t_{1}^{2} t_{2} t_{3}, t_{1} t_{2}^{2} t_{3}\right\}
$$

The differential $d: C^{4,3} \rightarrow C^{8,0}$ is given by $d\left(s_{1} t_{i} t_{j}\right)=a t_{i} t_{j}, d\left(s_{2} t_{i} t_{j}\right)=b t_{i} t_{j}$ and $d\left(s_{3} t_{i} t_{j}\right)=c t_{i} t_{j}$ when $i, j=1,2,3$. We calculate that

$$
\begin{aligned}
a t_{1}^{2} & =-2 t_{1}^{3} t_{2}+t_{1}^{2} t_{2}^{2}, \quad a t_{1} t_{2}=-3 t_{1}^{2} t_{2}^{2}, \quad a t_{1} t_{3}=t_{1}^{3} t_{3}-2 t_{1}^{2} t_{2} t_{3}+t_{1} t_{2}^{2} t_{3}, \\
a t_{2}^{2} & =2 t_{1}^{3} t_{2}+3 t_{1}^{2} t_{2}^{2}, \quad a t_{2} t_{3}=-t_{1}^{3} t_{3}-3 t_{1} t_{2}^{2} t_{3}, \\
b t_{1}^{2} & =-t_{1}^{3} t_{2}-t_{1}^{3} t_{3}-3 t_{1}^{2} t_{2} t_{3}, \quad b t_{1} t_{2}=-t_{1}^{3} t_{2}-t_{1}^{2} t_{2}^{2}-t_{1}^{2} t_{2} t_{3}-3 t_{1} t_{2}^{2} t_{3}, \\
b t_{1} t_{3} & =t_{1}^{3} t_{2}+t_{1}^{2} t_{2}^{2}+3 t_{1}^{2} t_{2} t_{3}+3 t_{1} t_{2}^{2} t_{3}, \quad b t_{2}^{2}=t_{1}^{3} t_{2}+3 t_{1}^{3} t_{3}+3 t_{1}^{2} t_{2} t_{3}+2 t_{1} t_{2}^{2} t_{3}, \\
b t_{2} t_{3} & =-3 t_{1}^{3} t_{2}-3 t_{1}^{3} t_{3}-3 t_{1}^{2} t_{2} t_{3}, \\
c t_{1}^{2} & =-2 t_{1}^{3} t_{2}-3 t_{1}^{2} t_{2}^{2}, \quad c t_{1} t_{2}=t_{1}^{2} t_{2}^{2}, \quad c t_{1} t_{3}=-3 t_{1}^{3} t_{3}-2 t_{1}^{2} t_{2} t_{3}-3 t_{1} t_{2}^{2} t_{3}, \\
c t_{2}^{2} & =2 t_{1}^{3} t_{2}-t_{1}^{2} t_{2}^{2}, \quad c t_{2} t_{3}=3 t_{1}^{3} t_{3}+t_{1} t_{2}^{2} t_{3} .
\end{aligned}
$$

We can see that the kernel of $d$ is free and of rank 10, and the cokernel of $d$ is isomorphic to $\mathbb{Z} /(2)^{\oplus 2}$ by using elementary transformations of matrices. Hence $H^{8,0} \cong \mathbb{Z} /(2)^{\oplus 2}$ and $H^{4,3} \cong \mathbb{Z}^{\oplus 7}$.

We have

$$
C^{2,9}=\mathbb{Z}\left\{s_{1} s_{2} s_{3}\right\} \otimes \mathbb{Z}\left\{t_{1}, t_{2}, t_{3}\right\}
$$

and

$$
C^{6,6}=\mathbb{Z}\left\{s_{1} s_{2}, s_{1} s_{3}, s_{2} s_{3}\right\} \otimes \mathbb{Z}\left\{t_{1}^{3}, t_{1}^{2} t_{2}, t_{1}^{2} t_{3}, t_{1} t_{2}^{2}, t_{1} t_{2} t_{3}, t_{2}^{2} t_{3}\right\} .
$$

The differential $d: C^{2,9} \rightarrow C^{6,6}$ is given by

$$
d\left(s_{1} s_{2} s_{3} t_{i}\right)=\left(a s_{2} s_{3}-b s_{1} s_{3}+c s_{1} s_{2}\right) t_{i}
$$


when $i=1,2,3$. We can easily see that $d$ is injective and the image of $d$ is a direct summand of $C^{6,6}$. Hence $H^{2,9}=\{0\}$.

We have

$$
C^{10,3}=\mathbb{Z}\left\{s_{1}, s_{2}, s_{3}\right\} \otimes \mathbb{Z}\left\{t_{1}^{3} t_{2}^{2}, t_{1}^{3} t_{2} t_{3}, t_{1}^{2} t_{2}^{2} t_{3}\right\} .
$$

The differential $d: C^{6,6} \rightarrow C^{10,3}$ is given by

$$
\begin{aligned}
& d\left(s_{1} s_{2} t_{i} t_{j} t_{k}\right)=\left(a s_{2}-b s_{1}\right) t_{i} t_{j} t_{k}, \\
& d\left(s_{1} s_{3} t_{i} t_{j} t_{k}\right)=\left(a s_{3}-c s_{1}\right) t_{i} t_{j} t_{k}, \\
& d\left(s_{2} s_{3} t_{i} t_{j} t_{k}\right)=\left(b s_{3}-c s_{2}\right) t_{i} t_{j} t_{k}
\end{aligned}
$$

when $i, j, k=1,2,3$. We calculate that

$$
\begin{aligned}
a t_{1}^{3} & =t_{1}^{3} t_{2}^{2}, \quad a t_{1}^{2} t_{2}=-3 t_{1}^{3} t_{2}^{2}, \quad a t_{1}^{2} t_{3}=-2 t_{1}^{3} t_{2} t_{3}+t_{1}^{2} t_{2}^{2} t_{3}, \\
a t_{1} t_{2}^{2} & =3 t_{1}^{3} t_{2}^{2}, \quad a t_{1} t_{2} t_{3}=-3 t_{1}^{2} t_{2}^{2} t_{3}, \quad a t_{2}^{2} t_{3}=2 t_{1}^{3} t_{2} t_{3}+3 t_{1}^{2} t_{2}^{2} t_{3}, \\
b t_{1}^{3} & =-3 t_{1}^{3} t_{2} t_{3}, \quad b t_{1}^{2} t_{2}=-t_{1}^{3} t_{2}^{2}-t_{1}^{3} t_{2} t_{3}-3 t_{1}^{2} t_{2}^{2} t_{3}, \quad b t_{1}^{2} t_{3}=t_{1}^{3} t_{2}^{2}+3 t_{1}^{3} t_{2} t_{3}+3 t_{1}^{2} t_{2}^{2} t_{3}, \\
b t_{1} t_{2}^{2} & =3 t_{1}^{3} t_{2} t_{3}+2 t_{1}^{2} t_{2}^{2} t_{3}, \quad b t_{1} t_{2} t_{3}=-3 t_{1}^{3} t_{2} t_{3}, \quad b t_{2}^{2} t_{3}=-3 t_{1}^{3} t_{2}^{2}-3 t_{1}^{3} t_{2} t_{3}-3 t_{1}^{2} t_{2}^{2} t_{3}, \\
c t_{1}^{3} & =-3 t_{1}^{3} t_{2}^{2}, \quad c t_{1}^{2} t_{2}=t_{1}^{3} t_{2}^{2}, \quad c t_{1}^{2} t_{3}=-2 t_{1}^{3} t_{2} t_{3}-3 t_{1}^{2} t_{2}^{2} t_{3}, \quad c t_{1} t_{2}^{2}=-t_{1}^{3} t_{2}^{2}, \\
c t_{1} t_{2} t_{3} & =t_{1}^{2} t_{2}^{2} t_{3}, \quad c t_{2}^{2} t_{3}=2 t_{1}^{3} t_{2} t_{3}-t_{1}^{2} t_{2}^{2} t_{3} .
\end{aligned}
$$

Then we can verify that the kernel of $d$ is free and of rank 9 , and the cokernel of $d$ is isomorphic to $\mathbb{Z} /(2)^{\oplus 2} \oplus \mathbb{Z} /(4)$ by using elementary transformations of matrices. Hence

$$
H^{10,3} \cong \mathbb{Z} /(2)^{\oplus 2} \oplus \mathbb{Z} /(4)
$$

and $H^{6,6} \cong \mathbb{Z}^{\oplus 6}$.

We have

$$
C^{0,9}=\mathbb{Z}\left\{s_{1} s_{2} s_{3}\right\}
$$

and

$$
C^{4,6}=\mathbb{Z}\left\{s_{1} s_{2}, s_{1} s_{3}, s_{2} s_{3}\right\} \otimes \mathbb{Z}\left\{t_{1}^{2}, t_{1} t_{2}, t_{1} t_{3}, t_{2}^{2}, t_{2} t_{3}\right\} .
$$

The differential $d: C^{0,9} \rightarrow C^{4,6}$ is given by

$$
d\left(s_{1} s_{2} s_{3}\right)=a s_{2} s_{3}-b s_{1} s_{3}+c s_{1} s_{2} .
$$

We can easily see that $d$ is injective and the image of $d$ is a direct summand of $C^{4,6}$. Hence $H^{0,9}=\{0\}$.

We have

$$
C^{8,3}=\mathbb{Z}\left\{s_{1}, s_{2}, s_{3}\right\} \otimes \mathbb{Z}\left\{t_{1}^{3} t_{2}, t_{1}^{3} t_{3}, t_{1}^{2} t_{2}^{2}, t_{1}^{2} t_{2} t_{3}, t_{1} t_{2}^{2} t_{3}\right\}
$$

The differential $d: C^{4,6} \rightarrow C^{8,3}$ is given by

$$
\begin{aligned}
& d\left(s_{1} s_{2} t_{i} t_{j}\right)=\left(a s_{2}-b s_{1}\right) t_{i} t_{j}, \\
& d\left(s_{1} s_{3} t_{i} t_{j}\right)=\left(a s_{3}-c s_{1}\right) t_{i} t_{j}, \\
& d\left(s_{2} s_{3} t_{i} t_{j}\right)=\left(b s_{3}-c s_{2}\right) t_{i} t_{j}
\end{aligned}
$$


when $i, j=1,2,3$. We can verify that the kernel of $d$ is free and of rank 3 , and the cokernel of $d$ is isomorphic to

$$
\mathbb{Z}^{\oplus 3} \oplus \mathbb{Z} /(2)^{\oplus 4} \oplus \mathbb{Z} /(8)^{\oplus 2}
$$

by using elementary transformations of matrices. Hence $H^{4,6} \cong \mathbb{Z}^{\oplus 2}$. By Lemma 4.5 we see that $d: C^{8,3} \rightarrow C^{12,0}$ is surjective and its kernel is free and of rank 14 . Hence

$$
H^{8,3} \cong \mathbb{Z}^{\oplus 2} \oplus \mathbb{Z} /(2)^{\oplus 4} \oplus \mathbb{Z} /(8)^{\oplus 2}
$$

Other groups $H^{p, q}$ can be computed by Lemma 4.5. By the universal coefficient theorem, $E_{5}^{10,0}=E_{5}^{12,0}=\{0\}$ for any $R$, and we see that the spectral sequence collapses from the $E_{5}$ term for any $R$ for reasons of degree. By sparseness, for every $n$ there is only one nontrivial $H^{p, q}$ whose total degree is given by $p+q=n$. Hence $H^{*}\left(Y_{R} ; \mathbb{Z}\right) \cong H$ as a graded module. This completes the proof.

Proposition 4.16. When $(n, m)=(4,4)$, the spectral sequence $E_{r}^{* * *}(Y)$ collapses from the $E_{7}$ term for any $R$. If $R=\mathbb{Z}$, then the $E_{\infty}$ term $H^{* * *}\left(C_{4}(4)\right)$ is given as follows.

\begin{tabular}{c|ccccccc}
15 & 0 & 0 & 0 & $\square^{3}$ & $\square^{5}$ & $\square^{3}$ & $\square$ \\
10 & 0 & 0 & $\square^{6}$ & $\square^{14} \oplus(2)$ & $\square^{12} \oplus(2)^{3}$ & $\square^{4} \oplus(2)^{5}$ & $(2)^{3}$ \\
5 & 0 & $\square^{4}$ & $\square^{12}$ & $\square^{14} \oplus(2)^{3}$ & $\square^{6} \oplus(2)^{7} \oplus(4)^{2}$ & $(2)^{7} \oplus(4)^{2}$ & $(2)^{3}$ \\
0 & $\square$ & $\square^{3}$ & $\square^{5}$ & $\square^{3} \oplus(2)^{3}$ & $(2)^{5}$ & $(2)^{3}$ & $(2)$ \\
\hline $9 / p$ & 0 & 2 & 4 & 6 & 8 & 10 & 12
\end{tabular}

The cohomology group $H^{*}\left(Y_{R}\right)$ is isomorphic to $H^{*}\left(C_{4}(4)\right)$ as a graded module.

Proof. For reasons of degree, the spectral sequence collapses from the $E_{7}$ term for any $R$. We write $C=C_{4}(4)$ and $H=H\left(C_{4}(4)\right)$, and set

$$
\begin{aligned}
& a=t_{1}^{3}-t_{1}^{2} t_{2}+2 t_{1} t_{2}^{2}, \\
& b=t_{1}^{3}-t_{1}^{2} t_{2}-t_{1} t_{2}^{2}-2 t_{1} t_{2} t_{3}-3 t_{2}^{2} t_{3}, \\
& c=-t_{1}^{2} t_{2}-3 t_{1}^{2} t_{3}-t_{1} t_{2}^{2}-2 t_{1} t_{2} t_{3}-3 t_{2}^{2} t_{3} .
\end{aligned}
$$

Then the submodule of $C^{6,0}$ generated by $a, b$ and $c$ is a direct summand of rank three. The differential $d: C^{0,5} \rightarrow C^{6,0}$ is given by $d\left(s_{1}\right)=2 a, d\left(s_{2}\right)=2 b$ and $d\left(s_{3}\right)=2 c$. Hence $H^{6,0} \cong \mathbb{Z}^{\oplus 3} \oplus \mathbb{Z} /(2)^{\oplus 3}$ and $H^{0,5}=\{0\}$.

We have

$$
C^{2,5}=\mathbb{Z}\left\{s_{1}, s_{2}, s_{3}\right\} \otimes \mathbb{Z}\left\{t_{1}, t_{2}, t_{3}\right\}
$$

and

$$
C^{8,0}=\mathbb{Z}\left\{t_{1}^{3} t_{2}, t_{1}^{3} t_{3}, t_{1}^{2} t_{2}^{2}, t_{1}^{2} t_{2} t_{3}, t_{1} t_{2}^{2} t_{3}\right\}
$$


We can calculate that

$$
\begin{aligned}
& a t_{1}=-t_{1}^{3} t_{2}+2 t_{1}^{2} t_{2}^{2}, \quad a t_{2}=-t_{1}^{3} t_{2}-3 t_{1}^{2} t_{2}^{2}, \quad a t_{3}=t_{1}^{3} t_{3}-t_{1}^{2} t_{2} t_{3}+2 t_{1} t_{2}^{2} t_{3}, \\
& b t_{1}=-t_{1}^{3} t_{2}-t_{1}^{2} t_{2}^{2}-2 t_{1}^{2} t_{2} t_{3}-3 t_{1} t_{2}^{2} t_{3}, \quad b t_{2}=2 t_{1}^{3} t_{2}+3 t_{1}^{3} t_{3}+3 t_{1}^{2} t_{2} t_{3}+t_{1} t_{2}^{2} t_{3}, \\
& b t_{3}=-3 t_{1}^{3} t_{2}-2 t_{1}^{3} t_{3}-2 t_{1}^{2} t_{2} t_{3}+t_{1} t_{2}^{2} t_{3}, \\
& c t_{1}=-t_{1}^{3} t_{2}-3 t_{1}^{3} t_{3}-t_{1}^{2} t_{2}^{2}-2 t_{1}^{2} t_{2} t_{3}-3 t_{1} t_{2}^{2} t_{3}, \quad c t_{2}=t_{1}^{3} t_{2}+3 t_{1}^{3} t_{3}+t_{1} t_{2}^{2} t_{3}, \\
& c t_{3}=3 t_{1}^{2} t_{2}^{2}+t_{1}^{2} t_{2} t_{3}+t_{1} t_{2}^{2} t_{3} .
\end{aligned}
$$

Then $C^{8,0}$ is generated by $a t_{i}, b t_{i}$ and $c t_{i}$, where $i=1,2,3$. Furthermore, the differential $d: C^{2,5} \rightarrow C^{8,0}$ is given by $d\left(s_{1} t_{i}\right)=2 a t_{i}, d\left(s_{2} t_{i}\right)=2 b t_{i}$ and $d\left(s_{3} t_{i}\right)=2 c t_{i}$ when $i=$ $1,2,3$. Hence $H^{8,0} \cong \mathbb{Z} /(2)^{\oplus 5}$ and $H^{2,5} \cong \mathbb{Z}^{\oplus 4}$.

We also have

$$
C^{4,5}=\mathbb{Z}\left\{s_{1}, s_{2}, s_{3}\right\} \otimes \mathbb{Z}\left\{t_{1}^{2}, t_{1} t_{2}, t_{1} t_{3}, t_{2}^{2}, t_{2} t_{3}\right\}
$$

and

$$
C^{10,0}=\mathbb{Z}\left\{t_{1}^{3} t_{2}^{2}, t_{1}^{3} t_{2} t_{3}, t_{1}^{2} t^{2} t_{3}\right\}
$$

The differential $d: C^{4,5} \rightarrow C^{10,0}$ is given by $d\left(s_{1} t_{i} t_{j}\right)=2 a t_{i} t_{j}, d\left(s_{2} t_{i} t_{j}\right)=2 b t_{i} t_{j}$ and $d\left(s_{3} t_{i} t_{j}\right)=2 c t_{i} t_{j}$ when $i, j=1,2,3$. We can calculate that

$$
\begin{aligned}
a t_{1}^{2} & =2 t_{1}^{3} t_{2}^{2}, \quad a t_{1} t_{2}=-3 t_{1}^{3} t_{2}^{2}, \quad a t_{1} t_{3}=-t_{1}^{3} t_{2} t_{3}+2 t_{1}^{2} t_{2}^{2} t_{3}, \\
a t_{2}^{2} & =2 t_{1}^{3} t_{2}^{2}, \quad a t_{2} t_{3}=-t_{1}^{3} t_{2} t_{3}-3 t_{1}^{2} t_{2}^{2} t_{3}, \\
b t_{1}^{2} & =-t_{1}^{3} t_{2}^{2}-2 t_{1}^{3} t_{2} t_{3}-3 t_{1}^{2} t_{2}^{2} t_{3}, \quad b t_{1} t_{2}=3 t_{1}^{3} t_{2} t_{3}+t_{1}^{2} t_{2}^{2} t_{3}, \\
b t_{1} t_{3} & =-2 t_{1}^{3} t_{2} t_{3}+t_{1}^{2} t_{2}^{2} t_{3}, \quad b t_{2}^{2}=2 t_{1}^{3} t_{2}^{2}+2 t_{1}^{3} t_{2} t_{3}+2 t_{1}^{2} t_{2}^{2} t_{3}, \\
b t_{2} t_{3} & =-3 t_{1}^{3} t_{2}^{2}-3 t_{1}^{3} t_{2} t_{3}-3 t_{1}^{2} t_{2}^{2} t_{3}, \\
c t_{1}^{2} & =-t_{1}^{3} t_{2}^{2}-2 t_{1}^{3} t_{2} t_{3}-3 t_{1}^{2} t_{2}^{2} t_{3}, \quad c t_{1} t_{2}=t_{1}^{2} t_{2}^{2} t_{3}, \\
c t_{1} t_{3} & =3 t_{1}^{3} t_{2}^{2}+t_{1}^{3} t_{2} t_{3}+t_{1}^{2} t_{2}^{2} t_{3}, \quad c t_{2}^{2}=t_{1}^{3} t_{2}^{2}+2 t_{1}^{3} t_{2} t_{3}-t_{1}^{2} t_{2}^{2} t_{3}, \quad c t_{2} t_{3}=-3 t_{1}^{3} t_{2}^{2}-t_{1}^{3} t_{2} t_{3} .
\end{aligned}
$$

Then we can see that $C^{10,0}$ is generated by $a t_{i} t_{j}, b t_{i} t_{j}$ and $c t_{i} t_{j}$, where $i, j=1,2,3$. Hence $H^{4,5} \cong \mathbb{Z}^{\oplus 12}$ and $H^{10,0} \cong \mathbb{Z} /(2)^{\oplus 3}$.

Now note that $C^{12,0} \cong \mathbb{Z}\left\{t_{1}^{3} t_{2}^{2} t_{3}\right\}$. It is easy to see that the image of the differential $d: C^{6,5} \rightarrow C^{12,0}$ is generated by $2 t_{1}^{3} t_{2}^{2} t_{3}$. Hence $H^{12,0} \cong \mathbb{Z} /(2)$. Note that the differential $d: C^{0,10} \rightarrow C^{6,5}$ is given by

$$
d\left(s_{1} s_{2}\right)=2\left(s_{2} a-s_{1} b\right), \quad d\left(s_{1} s_{3}\right)=2\left(s_{3} a-s_{1} c\right), \quad d\left(s_{2} s_{3}\right)=2\left(s_{3} b-s_{2} c\right) .
$$

We can easily see that $d$ is injective and so $H^{0,10}=\{0\}$. We can verify that the submodule of $C^{6,5}$ generated by $s_{2} a-s_{1} b, s_{3} a-s_{1} c$ and $s_{3} b-s_{2} c$ is a direct summand of the group of cycles by using elementary transformations of matrices. Hence $H^{6,5} \cong \mathbb{Z}^{\oplus 14} \oplus \mathbb{Z} /(2)^{\oplus 3}$.

Finally

$$
C^{4,10}=\mathbb{Z}\left\{s_{1} s_{2}, s_{1} s_{3}, s_{2} s_{3}\right\} \otimes \mathbb{Z}\left\{t_{1}^{2}, t_{1} t_{2}, t_{1} t_{3}, t_{2}^{2}, t_{2} t_{3}\right\}
$$


and

$$
C^{10,5}=\mathbb{Z}\left\{s_{1}, s_{2}, s_{3}\right\} \otimes \mathbb{Z}\left\{t_{1}^{3} t_{2}^{2}, t_{1}^{3} t_{2} t_{3}, t_{2}^{2} t_{2}^{2} t_{3}\right\} .
$$

The differential $d: C^{4,10} \rightarrow C^{10,5}$ is given by

$$
\begin{aligned}
& d\left(s_{1} s_{2} t_{i} t_{j}\right)=2\left(s_{2} a-s_{1} b\right) t_{i} t_{j}, \quad d\left(s_{1} s_{3} t_{i} t_{j}\right)=2\left(s_{3} a-s_{1} c\right) t_{i} t_{j}, \\
& d\left(s_{2} s_{3} t_{i} t_{j}\right)=2\left(s_{3} b-s_{2} c\right) t_{i} t_{j}
\end{aligned}
$$

where $i, j=1,2,3$. We can verify that the cokernel of $d$ is isomorphic to

$$
\mathbb{Z} /(2)^{7} \oplus \mathbb{Z} /(4)^{\oplus 2}
$$

using elementary matrix transformations. Hence $H^{4,10} \cong \mathbb{Z}^{\oplus 6}$ and

$$
H^{10,5} \cong \mathbb{Z} /(2)^{7} \oplus \mathbb{Z} /(4)^{\oplus 2} .
$$

Other groups $H^{p, q}$ can be computed by Lemma 4.5. By sparseness, for every $n$ there is only one nontrivial $H^{p, q}$ whose total degree is given by $p+q=n$. Hence $H^{*}\left(Y_{R} ; \mathbb{Z}\right) \cong H$ as a graded module. This completes the proof.

Proposition 4.17. When $(n, m)=(4,5)$, the spectral sequence $E_{r}^{*, *}(Y)$ collapses from the $E_{9}$ term for any $R$. If $R=\mathbb{Z}$, then the $E_{\infty}$ term $H^{*, *}\left(C_{4}(5)\right)$ is given as follows.

\begin{tabular}{c|ccccccc}
21 & 0 & 0 & $\square^{3}$ & $\square^{6}$ & $\square^{5}$ & $\square^{3}$ & $\square$ \\
14 & 0 & $\square^{3}$ & $\square^{12}$ & $\square^{18}$ & $\square^{14} \oplus(10)$ & $\square^{6} \oplus(10)^{3}$ & $\square \oplus(10)^{2}$ \\
7 & $\square$ & $\square^{6}$ & $\square^{14}$ & $\square^{18}$ & $\square^{12} \oplus(10)^{3}$ & $\square^{3} \oplus(10)^{6}$ & ${(10)^{3}}^{3}$ \\
0 & $\square$ & $\square^{3}$ & $\square^{5}$ & $\square^{6}$ & $\square^{3} \oplus(10)^{2}$ & ${(10)^{3}}^{3}$ & $(10)$ \\
\hline 9 & 0 & 2 & 4 & 6 & 8 & 10 & 12
\end{tabular}

The cohomology group $H^{*}\left(Y_{R}\right)$ is isomorphic to $H^{*}\left(C_{4}(5)\right)$ as a graded module.

Proof. For reasons of degree, the spectral sequence collapses from the $E_{9}$ term for any choice of $R$. We write $C=C_{4}(5)$ and $H=H\left(C_{4}(5)\right)$, and set $a=t_{1}^{2} t_{2}^{2}$ and $b=t_{1}^{3} t_{2}+t_{1}^{3} t_{3}+t_{1}^{2} t_{2} t_{3}$. We have $C^{0,7}=\mathbb{Z}\left\{s_{1}, s_{2}, s_{3}\right\}$ and $C^{8,0}=\mathbb{Z}\left\{a, b, t_{1}^{3} t_{2}, t_{1}^{3} t_{3}, t_{1} t_{2}^{2} t_{3}\right\}$. The differential $d: C^{0,7} \rightarrow C^{8,0}$ is given by $d\left(s_{1}\right)=10 a, d\left(s_{2}\right)=10 b$ and $d\left(s_{3}\right)=10 a$. Hence $H^{0,7}=\mathbb{Z}\left\{s_{1}-s_{3}\right\}$ and $H^{8,0} \cong \mathbb{Z}^{\oplus 3} \oplus \mathbb{Z} /(10)^{\oplus 2}$.

Now set $c=t_{1}^{3} t_{2}^{2}, d=t_{1}^{3} t_{2} t_{3}$ and $e=t_{1}^{2} t_{2}^{2} t_{3}$. We have

$$
C^{2,7}=\mathbb{Z}\left\{s_{1}, s_{2}, s_{3}\right\} \otimes \mathbb{Z}\left\{t_{1}, t_{2}, t_{3}\right\}
$$

and $C^{10,0}=\mathbb{Z}\{c, d, e\}$. The differential $d: C^{2,7} \rightarrow C^{10,0}$ is given by

$$
\begin{aligned}
& d\left(s_{1} t_{1}\right)=-d\left(s_{1} t_{2}\right)=d\left(s_{3} t_{1}\right)=-d\left(s_{3} t_{2}\right)=10 c, \\
& d\left(s_{1} t_{3}\right)=d\left(s_{3} t_{3}\right)=10 e, \quad d\left(s_{2} t_{1}\right)=10 d, \\
& d\left(s_{2} t_{2}\right)=-d\left(s_{2} t_{3}\right)=10(c+d+e) .
\end{aligned}
$$

Hence $H^{2,7} \cong \mathbb{Z}^{\oplus 6}$ and $H^{10,0} \cong \mathbb{Z} /(10)^{\oplus 3}$. 
Next set $f=t_{1}^{3} t_{2}^{2} t_{3}$. Then we have

$$
C^{4,7}=\mathbb{Z}\left\{t_{1}^{2}, t_{1} t_{2}, t_{1} t_{3}, t_{2}^{2}, t_{2} t_{3}\right\} \otimes \mathbb{Z}\left\{s_{1}, s_{2}, s_{3}\right\}
$$

and $C^{12,0}=\mathbb{Z}\{f\}$. The differential $d: C^{4,7} \rightarrow C^{12,0}$ is given by

$$
\begin{aligned}
d\left(s_{1} t_{1}^{2}\right) & =d\left(s_{1} t_{1} t_{2}\right)=d\left(s_{1} t_{2}^{2}\right)=d\left(s_{2} t_{1}^{2}\right)=d\left(s_{2} t_{2}^{2}\right) \\
& =d\left(s_{2} t_{2} t_{3}\right)=d\left(s_{3} t_{1}^{2}\right)=d\left(s_{3} t_{1} t_{2}\right)=d\left(s_{3} t_{2}^{2}\right) \\
& =0
\end{aligned}
$$

and

$$
d\left(s_{1} t_{1} t_{3}\right)=-d\left(s_{1} t_{2} t_{3}\right)=d\left(s_{2} t_{1} t_{2}\right)=-d\left(s_{2} t_{1} t_{3}\right)=d\left(s_{3} t_{1} t_{3}\right)=-d\left(s_{3} t_{2} t_{3}\right)=10 f .
$$

Hence $H^{4,7} \cong \mathbb{Z}^{\oplus 14}$ and $H^{12,0}=\mathbb{Z} /(10)\{f\}$.

We have $C^{0,14}=\mathbb{Z}\left\{s_{1} s_{2}, s_{1} s_{3}, s_{2} s_{3}\right\}$ and

$$
C^{8,7}=\mathbb{Z}\left\{s_{1}, s_{2}, s_{3}\right\} \otimes \mathbb{Z}\left\{a, b, t_{1}^{3} t_{2}, t_{1}^{3} t_{3}, t_{1} t_{2}^{2} t_{3}\right\} .
$$

The differential $d: C^{0,14} \rightarrow C^{8,7}$ is given by

$$
d\left(s_{1} s_{2}\right)=10\left(s_{2} a-s_{1} b\right), \quad d\left(s_{1} s_{3}\right)=10\left(s_{3}-s_{1}\right) a, \quad d\left(s_{2} s_{3}\right)=10\left(s_{3} b-s_{2} a\right) .
$$

Hence $H^{0,14}=\{0\}$ and $H^{8,7} \cong \mathbb{Z}^{\oplus 12} \oplus \mathbb{Z} /(10)^{\oplus 3}$.

We also have

$$
C^{2,14}=\mathbb{Z}\left\{s_{1} s_{2}, s_{1} s_{3}, s_{2} s_{3}\right\} \otimes \mathbb{Z}\left\{t_{1}, t_{2}, t_{3}\right\}
$$

and

$$
C^{10,7}=\mathbb{Z}\left\{s_{1}, s_{2}, s_{3}\right\} \otimes \mathbb{Z}\{c, d, e\} .
$$

The differential $d: C^{2,14} \rightarrow C^{10,7}$ is given by

$$
\begin{aligned}
& d\left(s_{1} s_{2} t_{1}\right)=10\left(s_{2} c-s_{1} d\right), \quad d\left(s_{1} s_{2} t_{2}\right)=-10\left(s_{2} c+s_{1}(c+d+e)\right), \\
& d\left(s_{1} s_{2} t_{3}\right)=10\left(s_{2} e+s_{1}(c+d+e)\right), \quad d\left(s_{1} s_{3} t_{1}\right)=10\left(s_{3}-s_{1}\right) c, \\
& d\left(s_{1} s_{3} t_{2}\right)=-10\left(s_{3}-s_{1}\right) c, \quad d\left(s_{1} s_{3} t_{3}\right)=10\left(s_{3}-s_{1}\right) e, \\
& d\left(s_{2} s_{3} t_{1}\right)=10\left(s_{3} d-s_{2} c\right), \quad d\left(s_{2} s_{3} t_{2}\right)=10\left(s_{3}(c+d+e)+s_{2} c\right), \\
& d\left(s_{2} s_{3} t_{3}\right)=-10\left(s_{3}(c+d+e)+s_{2} e\right) .
\end{aligned}
$$

Then the image of $d$ is given by

$$
\begin{aligned}
& \mathbb{Z}\left\{10\left(s_{3}-s_{1}\right) c, 10\left(s_{3}-s_{1}\right) e, 10\left(s_{2} c-s_{1} d\right),\right. \\
& \left.10 s_{2}(e-c), 10\left(s_{3} d-s_{2} c\right), 10\left(s_{1}(c+d+e)+s_{2} c\right)\right\} .
\end{aligned}
$$

Hence $H^{2,14} \cong \mathbb{Z}^{\oplus 3}$ and

$$
H^{10,7} \cong \mathbb{Z}^{\oplus 3} \oplus \mathbb{Z} /(10)^{\oplus 6}
$$

Other groups $H^{p, q}$ can be computed by Lemma 4.5. By sparseness, for every $n$ there is only one nontrivial $H^{p, q}$ whose total degree is given by $p+q=n$. Hence $H^{*}\left(Y_{R} ; \mathbb{Z}\right) \cong H$ as a graded module. This completes the proof. 
Proposition 4.18. When $(n, m)=(4,6)$, the spectral sequence $E_{r}^{*, *}(Y)$ collapses from the $E_{11}$ term for any $R$. If $R=\mathbb{Z}$, then the $E_{\infty}$ term $H^{* * *}\left(C_{4}(6)\right)$ is given as follows.

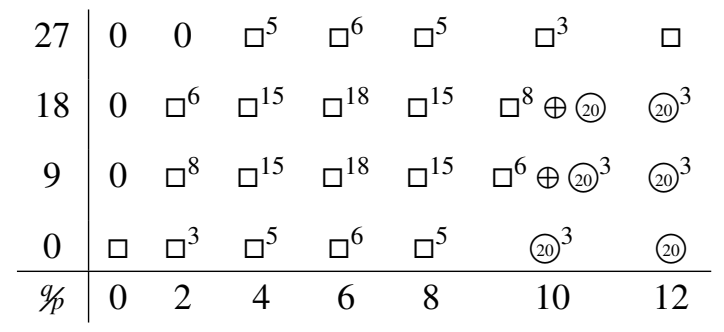

The cohomology groups $H^{*}\left(Y_{R}\right)$ and $H^{*}\left(C_{4}(6)\right)$ are isomorphic as graded modules.

Proof. For reasons of degree, the spectral sequence collapses from the $E_{11}$ term for any $R$. We write $C=C_{4}(6)$ and $H=H\left(C_{4}(6)\right)$, and set $a=t_{1}^{3} t_{2}^{2}, b=t_{1}^{3} t_{2}^{2}+t_{1}^{3} t_{2} t_{3}+t_{1}^{2} t_{2}^{2} t_{3}$, $c=t_{1}^{2} t_{2}^{2} t_{3}$ and $d=t_{1}^{3} t_{2}^{2} t_{3}$. Then $C^{10,0}=\mathbb{Z}\{a, b, c\}$ and $C^{12,0}=\mathbb{Z}\{d\}$. With respect to this basis, $d\left(s_{1}\right)=20 a, d\left(s_{2}\right)=20 b$ and $d\left(s_{3}\right)=20 c$. Hence $H^{10,0}=\mathbb{Z} /(20)\{a, b, c\}$ and $H^{0,9}=\{0\}$.

We also have

$$
C^{2,9}=\mathbb{Z}\left\{s_{1}, s_{2}, s_{3}\right\} \otimes \mathbb{Z}\left\{t_{1}, t_{2}, t_{3}\right\}
$$

Then

$$
d\left(s_{1} t_{1}\right)=d\left(s_{1} t_{2}\right)=d\left(s_{2} t_{2}\right)=d\left(s_{2} t_{3}\right)=d\left(s_{3} t_{3}\right)=0
$$

and

$$
d\left(s_{1} t_{3}\right)=d\left(s_{2} t_{1}\right)=d\left(s_{3} t_{1}\right)=-d\left(s_{3} t_{2}\right)=20 d .
$$

Hence $H^{12,0}=\mathbb{Z} /(20)\{d\}$ and $H^{2,9} \cong \mathbb{Z}^{\oplus 8}$.

Now note that $C^{0,18}=\mathbb{Z}\left\{s_{1} s_{2}, s_{1} s_{3}, s_{2} s_{3}\right\}$ and

$$
C^{10,9}=\mathbb{Z}\left\{s_{1}, s_{2}, s_{3}\right\} \otimes \mathbb{Z}\{a, b, c\} .
$$

The differential $d: C^{0,18} \rightarrow C^{10,9}$ is given by

$$
d\left(s_{1} s_{2}\right)=20\left(s_{2} a-s_{1} b\right), \quad d\left(s_{1} s_{3}\right)=20\left(s_{3} a-s_{1} c\right), \quad d\left(s_{2} s_{3}\right)=20\left(s_{3} b-s_{2} c\right) .
$$

Hence

$$
H^{10,9} \cong \mathbb{Z}^{\oplus 6} \oplus \mathbb{Z} /(20)^{\oplus 3}
$$

and $H^{0,18}=\{0\}$.

Other groups $H^{p, q}$ can be computed by Lemma 4.5. By sparseness, for every $n$ there is only one nontrivial $H^{p, q}$ whose total degree is given by $p+q=n$. Hence $H^{*}\left(Y_{R} ; \mathbb{Z}\right) \cong H$ as a graded module. This completes the proof.

When $(n, m)=(4,2),(4,3),(4,4),(4,5),(4,6)$ and $k$ is any field, we can obtain the Poincaré series of $Y_{R}$ from Remark 4.14, Propositions 4.15-4.18 and the universal coefficient theorem. 
Next we compare the spectral sequences for $E_{r}^{*, *}(Y)$ and $E_{r}^{*, *}(P)$.

LeMma 4.19. Let $m \geq 3$. For any commutative ring $R$ if $E_{r}^{* * *}(Y)$ collapses from the $E_{2 m-1}$ term, then $E_{r}^{*, *}(P)$ also collapses from the $E_{2 m-1}$ term.

Proof. We have the map of spectral sequences $E_{r}^{*, *}(P) \rightarrow E_{r}^{* * *}(Y)$. On the $E_{2 m-1}$ terms this map is given by

$$
\varepsilon \otimes 1: H^{*}\left(F_{4}\left(\mathbb{C}^{m}\right) ; R\right) \otimes_{R} H^{*}\left(C_{4}(m) ; R\right) \longrightarrow H^{*}\left(C_{4}(m) ; R\right)
$$

where $\varepsilon$ is the obvious augmentation. The next possible nontrivial differential in $E_{r}^{*, *}(P)$ is $d_{3(2 m-3)+1}$. Since $E_{2 m-1}^{*, 0}(P) \rightarrow E_{2 m-1}^{*, 0}(Y)$ is an isomorphism, the fact that $E_{r}^{*, *}(Y)$ collapses from the $E_{2 m-1}$ term implies that $E_{r}^{*, *}(P)$ also collapses from the $E_{2 m-1}$ term.

Corollary 4.20. When $n=4$ and $m \geq 3$, the sequence $E_{r}^{*, *}(P)$ collapses from the $E_{2 m-1}$ term.

Proof. This follows from Propositions 4.15-4.18 and Lemma 4.19.

Theorem 4.21. Let $R$ be a principal ideal domain. When $n=4$ and $m \geq 2$, there is an isomorphism of $H^{*}\left(F_{4}\left(\mathbb{C}^{m}\right) ; R\right)$-modules:

$$
H^{*}\left(\operatorname{Rep}_{4}(m)_{B} ; R\right) \cong H^{*}\left(F_{4}\left(\mathbb{C}^{m}\right) ; R\right) \otimes_{R} H^{*}\left(Y_{R} ; R\right) .
$$

The Poincaré series of $\operatorname{Rep}_{4}(m)_{B}$ is given by

$$
\operatorname{PS}\left(\operatorname{Rep}_{4}(m)_{B} ; k\right)=\operatorname{PS}\left(F_{4}\left(\mathbb{C}^{m}\right)\right) \cdot \operatorname{PS}\left(Y_{R} ; k\right)
$$

for any field $k$.

Proof. We may assume that $m \geq 3$ by Lemma 4.1 and Proposition 4.13. We have the map of spectral sequences $E_{r}^{*, *}(P) \rightarrow E_{r}^{*, *}(Y)$. By Propositions 4.15-4.18, $E_{r}^{*, *}(Y)$ collapses from the $E_{2 m-1}$ term. By Corollary $4.20, E_{r}^{* * *}(P)$ also collapses from the $E_{2 m-1}$ term. Note that $E_{2 m-1}^{*, 3(2 m-3)}(P)$ is free over $R$ since $R$ is a principal ideal domain. By comparing the $E_{\infty}$ terms we see that

$$
H^{*}\left(\operatorname{Rep}_{n}(m)_{B} ; R\right) \rightarrow H^{*}\left(Y_{R} ; R\right)
$$

is a split surjection of $R$-modules by Lemma 4.22 below. The theorem now follows by Proposition 4.4.

Lemma 4.22. Consider the following commutative diagram of $R$-modules:

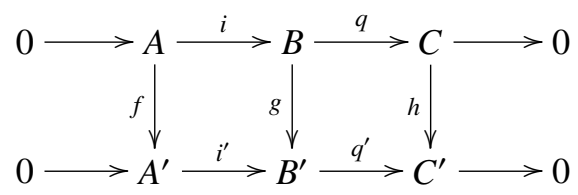

where the horizontal sequences are exact. If $f, h$ and $q$ are split surjective, then so is $g$. 
Proof. Let $a, c$ and $s$ be splitting maps of $f, h$ and $q$, respectively. Then $g s c q^{\prime}$ is an endomorphism of $B^{\prime}$ and $q^{\prime}=q^{\prime} g s c q^{\prime}$. Thus there is a homomorphism $k^{\prime}: B^{\prime} \rightarrow A^{\prime}$ such that $i^{\prime} k^{\prime}=1_{B^{\prime}}-g s c q^{\prime}$. We set $b=i a k^{\prime}+s c q^{\prime}$. Then $b$ is a homomorphism from $B^{\prime}$ to $B$ such that $g b=1_{B^{\prime}}$.

\section{The case when $m=2$}

In this section we deal with the case when $m=2$. The variety $\operatorname{Rep}_{n}(2)_{B}$ is in the unstable range and has different properties from $\operatorname{Rep}_{n}(m)_{B}$ when $m \geq 3$. For example, $H^{2}\left(\operatorname{Rep}_{n}(2)_{\mathrm{B}}\right)=\mathbb{Z} / n \mathbb{Z}$ by Corollary 5.3 and $\operatorname{Pic}\left(\operatorname{Rep}_{n}(2)_{B}\right)=\mathbb{Z} / n \mathbb{Z}$ by Proposition 5.9 . However, $H^{2}\left(\operatorname{Rep}_{n}(m)_{B}\right)=\mathbb{Z}^{n-1}$ if $m \geq 3$ by Proposition 5.5 .

Lemma 5.1. The space of $\mathbb{C}$-valued points of the character variety $\mathrm{Ch}_{n}(2)_{B}$ with classical topology is homotopy equivalent to $F_{n}\left(\mathbb{C}^{2}\right)$.

Proof. The lemma follows from the fact that there is a fibre bundle $\mathrm{Ch}_{n}(2)_{B} \rightarrow F_{n}\left(\mathbb{A}_{\mathbb{Z}}^{2}\right)$ with fibre $\mathbb{A}_{\mathbb{Z}}^{(n-2)(n-1) / 2}$ by [9, Proposition 3.8].

THEOREM 5.2. The space of $\mathbb{C}$-valued points of the representation variety $\operatorname{Rep}_{n}(2)_{B}$ with the classical topology is homotopy equivalent to $F_{n}\left(\mathbb{C}^{2}\right) \times \mathrm{PGL}_{n}(\mathbb{C})$.

Proof. For simplicity, let $\operatorname{Rep}_{n}(2)_{B}, \mathrm{~B}_{n}(2)_{B}, \mathrm{Ch}_{n}(2)_{B}, \mathrm{PGL}_{n}$ and $\mathrm{B}_{n}$ denote the spaces of $\mathbb{C}$-valued points of $\operatorname{Rep}_{n}(2)_{B}, \mathrm{~B}_{n}(2)_{B}, \mathrm{Ch}_{n}(2)_{B}, \mathrm{PGL}_{n}$ and $\mathrm{B}_{n}$ with the classical topology, respectively. Recall that

$$
\operatorname{Rep}_{n}(2)_{B}=\mathrm{B}_{n}(2)_{B} \times_{\mathrm{B}_{n}} \mathrm{PGL}_{n}
$$

and there is a fibre bundle

$$
\mathrm{PGL}_{n} \rightarrow \operatorname{Rep}_{n}(2)_{B} \rightarrow \mathrm{Ch}_{n}(2)_{B}
$$

This is a fibre bundle associated to the principal $\mathrm{B}_{n}$-bundle $\mathrm{B}_{n}(2)_{B} \rightarrow \mathrm{Ch}_{n}(2)_{B}$ with fibre $\mathrm{PGL}_{n}$.

The principal $\mathrm{B}_{n}$-bundle $\mathrm{B}_{n}(2)_{B} \rightarrow \mathrm{Ch}_{n}(2)_{B}$ is induced by a map $\mathrm{Ch}_{n}(2)_{B} \rightarrow B \mathrm{~B}_{n}$, where $B \mathrm{~B}_{n}$ is the classifying space of $\mathrm{B}_{n}$. By Lemma $5.1, \mathrm{Ch}_{n}(2)_{B} \simeq F_{n}\left(\mathbb{C}^{2}\right)$, which is 2-connected. The classifying space $B \mathrm{~B}_{n}$ is homotopy equivalent to the $(n-1)$-fold product of the Eilenberg-MacLane space $K(\mathbb{Z}, 2)$. Hence the map $\mathrm{Ch}_{n}(2)_{B} \rightarrow B \mathrm{~B}_{n}$ is homotopic to a constant map. This implies that the fibre bundle

$$
\mathrm{PGL}_{n} \rightarrow \operatorname{Rep}_{n}(2)_{B} \rightarrow \mathrm{Ch}_{n}(2)_{B}
$$

is trivial and

$$
\operatorname{Rep}_{n}(2)_{B} \simeq F_{n}\left(\mathbb{C}^{2}\right) \times \mathrm{PGL}_{n}
$$

as required. 
Corollary 5.3. We have $H^{2}\left(\operatorname{Rep}_{n}(2)_{B}\right) \cong \mathbb{Z} / n \mathbb{Z}$.

PRoof. This corollary follows from the facts that $H^{2}\left(\mathrm{PGL}_{n}(\mathbb{C})\right)=\mathbb{Z} / n \mathbb{Z}$ and $F_{n}\left(\mathbb{C}^{2}\right)$ is 2-connected.

Proposition 5.4. The rational cohomology ring of $\operatorname{Rep}_{n}(2)_{B}$ is given as follows:

$$
H^{*}\left(\operatorname{Rep}_{n}(2)_{B} ; \mathbb{Q}\right) \cong H^{*}\left(F_{n}\left(\mathbb{C}^{2}\right) ; \mathbb{Q}\right) \otimes \Lambda\left(e_{3}, \ldots, e_{2 n-1}\right),
$$

where $\left|e_{i}\right|=i$.

Proof. By Theorem 5.2, $\operatorname{Rep}_{n}(2)_{B}$ is homotopy equivalent to $F_{n}\left(\mathbb{C}^{2}\right) \times \mathrm{PGL}_{n}$. The proposition now follows from the fact that the homomorphism $\mathrm{SU}(n) \rightarrow \mathrm{PGL}_{n}$ induces an isomorphism on rational cohomology rings and $H^{*}(\mathrm{SU}(n)) \cong \Lambda\left(e_{3}, \ldots, e_{2 n-1}\right)$.

On the other hand, the cohomology group $H^{2}\left(\operatorname{Rep}_{n}(m)_{B}\right)$ is different when $m \geq 3$ from when $m=2$.

Proposition 5.5. If $m \geq 3$, then $H^{2}\left(\operatorname{Rep}_{n}(m)_{\mathrm{B}}\right)=\mathbb{Z}^{n-1}$.

PRoof. There is a fibration

$$
\mathrm{B}_{n}(m)_{B} \rightarrow \operatorname{Rep}_{n}(m)_{B} \rightarrow \operatorname{Flag}\left(\mathbb{C}^{n}\right) .
$$

If $m \geq 3$, then $\mathrm{B}_{n}(m)_{B}$ is 2-connected [9, Lemma 4.2]. Hence the map

$$
H^{2}\left(\operatorname{Flag}\left(\mathbb{C}^{n}\right)\right) \rightarrow H^{2}\left(\operatorname{Rep}_{n}(m)_{B}\right)
$$

is an isomorphism. Since

$$
H^{2}\left(\operatorname{Flag}\left(\mathbb{C}^{n}\right)\right)=\mathbb{Z}\left\{t_{1}, \ldots, t_{n}\right\} / \mathbb{Z}\left\{c_{1}\right\} \cong \mathbb{Z}^{n-1},
$$

the proposition now follows.

From now on, until the end of this section, we shall assume that all varieties are defined over $\mathbb{Z}$. The following results show that $\operatorname{Pic}\left(\operatorname{Rep}_{n}(2)_{B}\right)=\mathbb{Z} / n \mathbb{Z}$.

Lemma 5.6. Let $Y$ be a noetherian separated integral scheme that is regular in codimension one. Let $f: X \rightarrow Y$ be an $\mathbb{A}_{Z}^{n}$-fibre bundle with a local trivialization with respect to the Zariski topology. Then $X$ is also a noetherian separated integral scheme that is regular in codimension one. Furthermore, there is an isomorphism between divisor class groups $f^{*}: \mathrm{Cl}(Y) \stackrel{\cong}{\longrightarrow} \mathrm{Cl}(X)$.

Proof. This lemma can be proved in a similar way to [3, Ch. II, Proposition 6.6]. It is easy to see that $X$ is a noetherian separated integral scheme that is regular in codimension one.

The scheme $X$ has two types of codimension one points. If the image of a codimension one point by $f$ also has codimension one in $Y$, then call it type 1 . If the image is a generic point of $Y$, then call it type 2. Note that any type 1 point can be expressed as $f^{-1}(D)$ for some prime divisor $D$ in $Y$. 
We define a homomorphism $f^{*}: \mathrm{Cl}(Y) \rightarrow \mathrm{Cl}(X)$ by

$$
D=\sum n_{i} D_{i} \mapsto f^{*} D=\sum n_{i} f^{-1}\left(D_{i}\right)
$$

We can easily check that $f^{*}$ is well-defined. To show that $f^{*}$ is injective, suppose that $f^{*} D=(\varphi)$ in $\operatorname{Div} X$ for some divisor $D$ in $Y$ and some rational function $\varphi$ on $X$. Take a nonempty open subset $U$ of $Y$ such that $f^{-1}(U) \cong U \times \mathbb{A}_{\mathbb{Z}}^{n}$. The rational function $\varphi$ is expressed as a quotient $\psi / \xi$ on $f^{-1}(U)$ where

$$
\psi, \xi \in \mathcal{K}_{U} \otimes_{O_{U}} O_{U \times \mathbb{A}_{z}^{n}} \cong \mathcal{K}_{U}\left[x_{1}, \ldots, x_{n}\right] .
$$

If $\left.\varphi\right|_{U}$ is not contained in $\mathcal{K}_{U}$, then $(\varphi)$ contains a divisor of type 2 . However, $f^{*} D$ contains only divisors of type 1 . This is a contradiction. Thus $\left.\varphi\right|_{U} \in \mathcal{K}_{U}$ and hence $\varphi \in \mathcal{K}_{Y}$. This implies that $D=(\varphi)$ as a divisor on $Y$ and therefore $f^{*}$ is injective.

To show that $f^{*}$ is surjective, it suffices to prove that each prime divisor $D$ of type 2 is linearly equivalent to a sum of divisors of type 1 on $X$. Let $\eta$ be a generic point of $D$ and take $U$ as before. The point

$$
\eta \in f^{-1}(U) \cong U \times \mathbb{A}_{\mathbb{Z}}^{n}
$$

corresponds to a principal ideal $(\varphi) \subseteq \mathcal{K}_{U}\left[x_{1}, \ldots, x_{n}\right]$. The rational function $\varphi \in \mathcal{K}_{X}$ defines a divisor $D+\sum m_{i} D_{i}$ with prime divisors $D_{i}$ of type 1 . This implies that $D$ is linearly equivalent to a sum of divisors of type 1 , and hence $f^{*}$ is an isomorphism, as required.

By [9, Proposition 3.8], there is a fibre bundle $\mathrm{Ch}_{n}(m)_{B} \rightarrow F_{n}\left(\mathbb{A}_{\mathbb{Z}}^{m}\right)$ with fibre

$$
\left(\mathbb{P}_{\mathbb{Z}}^{m-2}\right)^{n-1} \times\left(\mathbb{A}_{\mathbb{Z}}^{m-1}\right)^{(n-2)(n-1) / 2} .
$$

If $m=2$, then $\mathrm{Ch}_{n}(2)_{B} \rightarrow F_{n}\left(\mathbb{A}_{\mathbb{Z}}^{2}\right)$ is an $\left(\mathbb{A}_{\mathbb{Z}}^{1}\right)^{(n-2)(n-1) / 2}$-fibre bundle. The next corollary follows from Lemma 5.6.

Corollary 5.7. We have $\mathrm{Cl}\left(\mathrm{Ch}_{n}(2)_{B}\right)=\operatorname{Pic}\left(\mathrm{Ch}_{n}(2)_{B}\right)=\{0\}$.

Proof. Lemma 5.6 implies that the map

$$
\mathrm{Cl}\left(F_{n}\left(\mathbb{A}_{\mathbb{Z}}^{m}\right)\right) \rightarrow \mathrm{Cl}\left(\mathrm{Ch}_{n}(2)_{B}\right)
$$

is an isomorphism. The configuration space $F_{n}\left(\mathbb{A}_{\mathbb{Z}}^{m}\right)$ is an open subscheme of the affine space $\left(\mathbb{A}_{\mathbb{Z}}^{m}\right)^{n}$ and hence $\mathrm{Cl}\left(F_{n}\left(\mathbb{A}_{\mathbb{Z}}^{m}\right)\right)=\{0\}$. Therefore

$$
\mathrm{Cl}\left(\mathrm{Ch}_{n}(2)_{B}\right)=\operatorname{Pic}\left(\mathrm{Ch}_{n}(2)_{B}\right)=\{0\},
$$

as required.

Lemma 5.8. Let $Y$ be a noetherian separated integral scheme that is regular in codimension one. Let $f: X \rightarrow Y$ be a $\mathrm{PGL}_{n}$-fibre bundle with a local trivialization with respect to the Zariski topology. Then $X$ is also a noetherian separated integral scheme that is regular in codimension one. Furthermore, if $\mathrm{Cl}(Y)=\{0\}$, then $\mathrm{Cl}(X)=\mathbb{Z} / n \mathbb{Z}$. 
Proof. In the same way as Lemma 5.6 we can prove that $X$ is a noetherian separated integral scheme that is regular in codimension one. In the present proof we also use the notion of divisors of types 1 and 2 on $X$ as in the proof of Lemma 5.6.

Let us prove that $\mathrm{Cl}(X)=\mathbb{Z} / n \mathbb{Z}$ if $\mathrm{Cl}(Y)=\{0\}$. Recall that $\mathrm{Cl}\left(\mathrm{PGL}_{n}\right)=\mathbb{Z} / n \mathbb{Z}$ because $\mathrm{PGL}_{n}$ is isomorphic to the complement of a closed subscheme of degree $n$ of $\mathbb{P}^{n^{2}-1}$. First we assume that $X \cong Y \times \mathrm{PGL}_{n}$. The scheme $Y \times \mathrm{PGL}_{n}$ is the complement of a closed subscheme of degree $n$ of $Y \times \mathbb{P}^{n^{2}-1}$. The surjective homomorphism

$$
\mathrm{Cl}\left(Y \times \mathbb{P}^{n^{2}-1}\right)=\mathrm{Cl}(Y) \times \mathbb{Z}=\mathbb{Z} \rightarrow \mathrm{Cl}\left(Y \times \mathrm{PGL}_{n}\right)
$$

induces an isomorphism $\mathrm{Cl}\left(Y \times \mathrm{PGL}_{n}\right) \cong \mathbb{Z} / n \mathbb{Z}$.

Next we deal with general $\mathrm{PGL}_{n}$-fibre bundles. Let $H$ be a prime divisor of $\mathrm{PGL}_{n}$ that is a generator of $\mathrm{Cl}\left(\mathrm{PGL}_{n}\right)=\mathbb{Z} / n \mathbb{Z}$. We take a nonempty open subset $U$ of $Y$ such that $f^{-1}(U) \cong U \times \mathrm{PGL}_{n}$ and define a homomorphism $\varphi: \mathbb{Z} / n \mathbb{Z} \rightarrow \mathrm{Cl}(X)$ by $\varphi([1])=\overline{U \times H}$, where $\overline{U \times H}$ denotes the closure of $U \times H \subseteq f^{-1}(U)$ in $X$. In order to verify that $\varphi$ is well-defined, it suffices to prove that $n(\overline{U \times H})$ is linearly equivalent to 0 . Since the divisor $n H$ on $\mathrm{PGL}_{n}$ is linearly equivalent to 0 , we have that $n(U \times H)=(g)$ for some rational function $g$ on $f^{-1}(U)$. The divisor $(g)$ on $X$ is equal to $n(\overline{U \times H})+\sum n_{i} D_{i}$, where the $D_{i}$ are divisors of type 1 . Each divisor $D_{i}$ of type 1 can be written in the form $f^{-1}\left(E_{i}\right)$ for some divisor $E_{i}$ on $Y$. By the hypothesis that $\mathrm{Cl}(Y)=\{0\}$ we have $D_{i}=f^{-1}\left(E_{i}\right) \sim 0$. Hence $n(\overline{U \times H}) \sim 0$.

We now show that $\varphi$ is an isomorphism. Any divisors of type 1 on $X$ are linearly equivalent to 0 because $\mathrm{Cl}(Y)=\{0\}$. Let $D$ be a prime divisor of type 2 . The divisor $\left.D\right|_{f^{-1}(U)}$ on $f^{-1}(U)$ is linearly equivalent to $m(U \times H)$ for some $m$ since $\mathrm{Cl}(U)=\{0\}$ implies that

$$
\mathrm{Cl}\left(f^{-1}(U)\right) \cong \mathrm{Cl}\left(U \times \mathrm{PGL}_{n}\right) \cong \mathbb{Z} / n \mathbb{Z} .
$$

Hence the divisor class $[D]$ is contained in Image $\varphi$. Therefore $\varphi$ is surjective. The inclusion $f^{-1}(U) \subseteq X$ induces a surjection

$$
\mathrm{Cl}(X) \rightarrow \mathrm{Cl}\left(f^{-1}(U)\right) \cong \mathbb{Z} / n \mathbb{Z},
$$

which implies that $\varphi$ is injective. We have thus proved that $\varphi$ is an isomorphism.

The quotient morphism $\operatorname{Rep}_{n}(2)_{B} \rightarrow \mathrm{Ch}_{n}(2)_{B}$ is a $\mathrm{PGL}_{n}$-fibre bundle that has a local trivialization with respect to the Zariski topology. We can deduce the following proposition from Corollary 5.7 and Lemma 5.8.

Proposition 5.9. We have

$$
\mathrm{Cl}\left(\operatorname{Rep}_{n}(2)_{B}\right)=\operatorname{Pic}\left(\operatorname{Rep}_{n}(2)_{B}\right)=\mathbb{Z} / n \mathbb{Z} .
$$

There is a universal action of the free monoid $\Upsilon_{2}$ of rank two on the trivial bundle $O_{\operatorname{Rep}_{n}(2)_{B}}^{n}$ on $\operatorname{Rep}_{n}(2)_{B}$. There exists a universal $\Upsilon_{2}$-stable flag

$$
\{0\}=\mathcal{L}_{0} \subset \mathcal{L}_{1} \subset \mathcal{L}_{2} \subset \cdots \subset \mathcal{L}_{n}=O_{\operatorname{Rep}_{n}(2)_{B}}^{n} .
$$


We shall determine the isomorphism classes of $\mathcal{L}_{1}, \mathcal{L}_{2} / \mathcal{L}_{1}, \ldots$, and $\mathcal{L}_{n} / \mathcal{L}_{n-1}$ in $\operatorname{Pic}\left(\operatorname{Rep}_{n}(2)_{B}\right)=\mathbb{Z} / n \mathbb{Z}$. We set $L_{i}:=\mathcal{L}_{i} / \mathcal{L}_{i-1}$ when $i=1,2, \ldots, n$.

LEMma 5.10. We have

$$
\mathrm{Cl}\left(\mathrm{B}_{n}(m)_{B}\right)=\operatorname{Pic}\left(\mathrm{B}_{n}(m)_{B}\right)=\{0\} .
$$

Proof. The scheme $\mathrm{B}_{n}(m)_{B}$ represents the $m$ upper triangular matrices that generate the algebra of upper triangular matrices. Hence $\mathrm{B}_{n}(m)_{B}$ is isomorphic to an open subscheme of $\left(\mathbb{A}_{\mathbb{Z}}^{n(n+1) / 2}\right)^{m}$. Since $\operatorname{Cl}\left(\left(\mathbb{A}_{\mathbb{Z}}^{n(n+1) / 2}\right)^{m}\right)=\{0\}$ we have $\operatorname{Cl}\left(\mathrm{B}_{n}(m)_{B}\right)=\{0\}$.

Corollary 5.11. We have

$$
\mathrm{Cl}\left(\mathrm{B}_{n}(m)_{B} \times \mathrm{PGL}_{n}\right)=\operatorname{Pic}\left(\mathrm{B}_{n}(m)_{B} \times \mathrm{PGL}_{n}\right)=\mathbb{Z} / n \mathbb{Z} .
$$

Proof. The statement follows from Lemmas 5.10 and 5.8.

By [9, Section 2.2], there is a $\mathrm{B}_{n}$-bundle

$$
\mathrm{B}_{n}(2)_{B} \times \mathrm{PGL}_{n} \rightarrow \operatorname{Rep}_{n}(2)_{B} .
$$

This morphism induces a map

$$
\psi: \mathrm{Cl}\left(\operatorname{Rep}_{n}(2)_{B}\right)=\mathbb{Z} / n \mathbb{Z} \rightarrow \mathrm{Cl}\left(\mathrm{B}_{n}(2)_{B} \times \mathrm{PGL}_{n}\right)=\mathbb{Z} / n \mathbb{Z} .
$$

We show that $\psi$ is an isomorphism. In order to prove this, we assume that each variety is defined over $\mathbb{C}$ for a while. By considering the universal flag we have $\operatorname{Rep}_{n}(2)_{B} \rightarrow \operatorname{Flag}\left(\mathbb{C}^{n}\right)$.

The composition of morphisms

$$
h: \mathrm{PGL}_{n}=\{*\} \times \mathrm{PGL}_{n} \rightarrow \mathrm{B}_{n}(2)_{B} \times \mathrm{PGL}_{n} \rightarrow \operatorname{Rep}_{n}(2)_{B} \rightarrow \operatorname{Flag}\left(\mathbb{C}^{n}\right)
$$

is the quotient morphism

$$
\mathrm{PGL}_{n} \rightarrow \mathrm{PGL}_{n} / \mathrm{B}_{n}=\operatorname{Flag}\left(\mathbb{C}^{n}\right) .
$$

Let

$$
\{0\} \subset \mathcal{L}_{1}^{\prime} \subset \mathcal{L}_{2}^{\prime} \subset \cdots \subset \mathcal{L}_{n}^{\prime}=\mathbb{C}^{n}
$$

denote the universal flag on $\operatorname{Flag}\left(\mathbb{C}^{n}\right)$ and let $t_{i}=c_{1}\left(\mathcal{L}_{i}^{\prime} / \mathcal{L}_{i-1}^{\prime}\right)$. In $H^{2}\left(\mathrm{PGL}_{n}\right) \cong \mathbb{Z} / n \mathbb{Z}$ we see that

$$
h^{*}\left(t_{1}\right)=h^{*}\left(t_{2}\right)=\cdots=h^{*}\left(t_{n}\right)
$$

and that $h^{*}\left(t_{1}\right)$ is a generator of $H^{2}\left(\mathrm{PGL}_{n}\right)$. Hence $\psi\left(L_{1}\right), \psi\left(L_{1}^{\otimes 2}\right), \ldots, \psi\left(L_{1}^{\otimes(n-1)}\right)$ and $O_{\mathrm{PGL}_{n}}$ give us distinct isomorphism classes of topological line bundles. This implies that $\psi$ is surjective and that $\psi$ is an isomorphism. Moreover,

$$
\psi\left(L_{1}\right) \cong \psi\left(L_{2}\right) \cong \cdots \cong \psi\left(L_{n}\right)
$$

since their pull-backs are not topologically isomorphic to any of the pull-backs of $\psi\left(L_{1}^{\otimes 2}\right), \ldots, \psi\left(L_{1}^{\otimes(n-1)}\right)$ and $O_{\mathrm{PGL}_{n}}$ on $\mathrm{PGL}_{n}$. Therefore we have the following theorem.

THEOREM 5.12. For the universal flag

$$
\{0\} \subset \mathcal{L}_{1} \subset \mathcal{L}_{2} \subset \cdots \subset \mathcal{L}_{n}=O_{\operatorname{Rep}_{n}(2)_{B}}^{n}
$$


on the variety $\operatorname{Rep}_{n}(2)_{B}$ over $\mathbb{Z}$, we set $L_{i}:=\mathcal{L}_{i} / \mathcal{L}_{i-1}$ when $i=1,2, \ldots, n$. Then $L_{1} \cong L_{2} \cong \cdots \cong L_{n}$. Furthermore, $L_{1}$ gives a generator of $\operatorname{Pic}\left(\operatorname{Rep}_{n}(2)_{B}\right) \cong \mathbb{Z} / n \mathbb{Z}$.

We also obtain the same theorem for groups or monoids generated by two elements. Lemma 5.13. Let $\Gamma=\langle a, b\rangle$ be a group generated by two elements $a$ and $b$. Let $\varphi: \Upsilon_{2}=\langle\alpha, \beta\rangle \rightarrow \Gamma$ be the monoid homomorphism defined by $\alpha \mapsto a$ and $\beta \mapsto b$. For $a$ representation $\rho$ of $\Gamma$ on a scheme $X$, we denote by $\rho \circ \varphi$ the representation of $\Upsilon_{2}$ on $X$ obtained by taking the composition of $\rho$ and $\varphi$. Then $\rho$ is a representation with Borel mold if and only if $\rho \circ \varphi$ is also a representation with Borel mold.

Proof. By the Cayley-Hamilton theorem, the statement follows from the fact that $\rho(a)^{-1}$ and $\rho(b)^{-1}$ are expressed by polynomials of $\rho(a)$ and $\rho(b)$ respectively.

THEOREM 5.14. Let $\Gamma$ be a group or a monoid generated by two elements. Let

$$
\{0\} \subset \mathcal{L}(\Gamma)_{1} \subset \mathcal{L}(\Gamma)_{2} \subset \cdots \subset \mathcal{L}(\Gamma)_{n}=O_{\operatorname{Rep}_{n}(\Gamma)_{B}}^{n}
$$

be the universal flag on the representation variety $\operatorname{Rep}_{n}(\Gamma)_{B}$ with Borel mold over $\mathbb{Z}$. Then

$$
\mathcal{L}(\Gamma)_{1} \cong \mathcal{L}(\Gamma)_{2} / \mathcal{L}(\Gamma)_{1} \cong \mathcal{L}(\Gamma)_{3} / \mathcal{L}(\Gamma)_{2} \cong \cdots \cong \mathcal{L}(\Gamma)_{n} / \mathcal{L}(\Gamma)_{n-1}
$$

and $\mathcal{L}(\Gamma)_{1}^{\otimes n} \cong \mathcal{O}_{X}$.

Proof. Let

$$
\varphi: \Upsilon_{2}=\langle\alpha, \beta\rangle \rightarrow \Gamma=\langle a, b\rangle
$$

be the monoid homomorphism defined by $\alpha \mapsto a, \beta \mapsto b$. The homomorphism $\varphi$ induces a morphism

$$
\hat{\varphi}: \operatorname{Rep}_{n}(\Gamma)_{B} \rightarrow \operatorname{Rep}_{m}(2)_{B}
$$

by Lemma 5.13. Then $\hat{\varphi}^{*}\left(\mathcal{L}_{i}\right)=\mathcal{L}(\Gamma)_{i}$. The result follows, by Theorem 5.12.

Corollary 5.15. Let $X$ be an affine scheme. Let $\rho$ be a representation with Borel mold of degree $n$ on $X$ for a group or a monoid $\Gamma$ generated by two elements. Suppose that Pic $X$ has no nontrivial $n$-torsion elements. Then $\rho$ has the unique $\Gamma$-stable flag

$$
\{0\} \subset O_{X} \subset O_{X}^{2} \subset \cdots \subset O_{X}^{n} .
$$

In other words, there exists a suitable matrix $P \in \mathrm{GL}_{n}(R)$ such that $P^{-1} \rho(\gamma) P$ is an upper triangular matrix for each $\gamma \in \Gamma$, where $R$ is the coordinate ring of $X$.

Proof. Let

$$
\{0\} \subset M_{1} \subset M_{2} \subset \cdots \subset M_{n}=O_{X}^{n}
$$

be the $\Gamma$-stable flag on $X$. By Theorem 5.14

$$
M_{1} \cong M_{2} / M_{1} \cong M_{3} / M_{2} \cong \cdots \cong M_{n} / M_{n-1}
$$

and $M_{1}^{\otimes n} \cong O_{X}$. Since Pic $X$ has no nontrivial $n$-torsion elements, $M_{1} \cong O_{X}$. The hypothesis that $X$ is affine implies that $M_{2}, M_{3}, \ldots, M_{n}$ are trivial. This completes the proof. 
We now discuss the relationship between representations with Borel mold and the ring of integers of quadratic fields. We can see some elementary number theory results from a different viewpoint as an application of representations with Borel mold.

Proposition 5.16. Let $R$ be a commutative ring. There exists a two-dimensional representation with Borel mold over $R$ for the free group $F_{2}$ of rank two if and only if there exist $a, b \in R^{\times}$such that $R(a-1)+R(b-1)=R$. If it holds, then there is no ring homomorphism from $R$ to the field $\mathbb{F}_{2}=\{0,1\}$.

Proof. Suppose that there exist $a, b \in R^{\times}$such that the condition

$$
R(a-1)+R(b-1)=R
$$

holds. We take $u, v \in R$ such that $(a-1) v-(b-1) u=1$ and define the twodimensional representation $\rho$ over $R$ for the free group $F_{2}=\langle\alpha, \beta\rangle$ of rank two by

$$
\rho(\alpha)=\left(\begin{array}{cc}
a & u \\
0 & 1
\end{array}\right), \quad \rho(\beta)=\left(\begin{array}{ll}
b & v \\
0 & 1
\end{array}\right) .
$$

It is easy to check that $\rho$ is a representation with Borel mold.

Assume that there exists a two-dimensional representation $\rho$ with Borel mold over $R$ for the free group $F_{2}$ of rank two. Let $L \subset R^{2}$ be the $\rho$-invariant line subbundle of $R^{2}$. Setting $M:=R^{2} / L$, we have $R^{2} \cong L \oplus M$. By Theorem 5.14 we see that $L^{\otimes 2} \cong M^{\otimes 2} \cong R$ and that $L \cong M$. Then we can regard $\rho$ as

$$
\rho(\gamma)=\left(\begin{array}{cc}
\rho_{11}(\gamma) & \rho_{12}(\gamma) \\
0 & \rho_{22}(\gamma)
\end{array}\right) \in\left(\begin{array}{cc}
\operatorname{Hom}(L, L) & \operatorname{Hom}(M, L) \\
\operatorname{Hom}(L, M) & \operatorname{Hom}(M, M)
\end{array}\right)=\left(\begin{array}{ll}
R & R \\
R & R
\end{array}\right)
$$

where $\gamma \in F_{2}$. We see that there exists a two-dimensional representation $\rho$ with Borel mold over $R$ if and only if there exists a two-dimensional representation $\rho^{\prime}: F_{2} \rightarrow$ $\mathcal{B}_{2}(R)$ with Borel mold. Here $\mathcal{B}_{2}(R)$ is the subalgebra of upper triangular matrices of $\mathrm{M}_{2}(R)$. Hence we may begin by assuming that $L \cong R$ and that $\rho: F_{2} \rightarrow \mathcal{B}_{2}(R)$.

Let

$$
\rho(\alpha)=\left(\begin{array}{cc}
a_{1} & a_{3} \\
0 & a_{2}
\end{array}\right) \quad \text { and } \rho(\beta)=\left(\begin{array}{cc}
b_{1} & b_{3} \\
0 & b_{2}
\end{array}\right) .
$$

Since we have assumed that $\rho$ is a representation with Borel mold, we obtain $\left(a_{1}-a_{2}\right) b_{3}-\left(b_{1}-b_{2}\right) a_{3} \in R^{\times}$. This implies that $R\left(a_{1}-a_{2}\right)+R\left(b_{1}-b_{2}\right)=R$. Since

$$
R\left(a_{1} a_{2}^{-1}-1\right)+R\left(b_{1} b_{2}^{-1}-1\right)=R
$$

condition (5.1) holds.

Assume that condition (5.1) holds. Suppose that there exists a ring homomorphism $R \rightarrow \mathbb{F}_{2}$. The reduction $\rho \otimes_{R} \mathbb{F}_{2}$ is also a representation with Borel mold. However, no representation with Borel mold over $\mathbb{F}_{2}$ exists because there is no $a, b \in\left(\mathbb{F}_{2}\right)^{\times}$such that

$$
\mathbb{F}_{2}(a-1)+\mathbb{F}_{2}(b-1)=\mathbb{F}_{2} .
$$

This is a contradiction. Hence there is no ring homomorphism $R \rightarrow \mathbb{F}_{2}$. 
Corollary 5.17. Let $R$ be the ring of integers of the quadratic field $\mathbb{Q}(\sqrt{m})$. Assume that there exist $a, b \in R^{\times}$such that $R(a-1)+R(b-1)=R$. Then 2 is prime in $R$. Moreover, $m \equiv 5 \bmod 8$.

Proof. Under the assumption, there is no ring homomorphism $R \rightarrow \mathbb{F}_{2}$. It follows that $2 R$ is a prime ideal of $R$. Hence $m \equiv 5 \bmod 8$ by elementary number theory (see [2, Ch. III, (2.29)], [12, Theorem 5.17]).

Let $R$ be the ring of integers of the quadratic field $\mathbb{Q}(\sqrt{m})$. We investigate the case for which there exist $a, b \in R^{\times}$such that

$$
R(a-1)+R(b-1)=R .
$$

By Corollary 5.17 we may assume that $m \equiv 5 \bmod 8$. Hence $R=\mathbb{Z}\left[\frac{1}{2}(1+\sqrt{m})\right]$ (see $[2$, Ch. II, (1.33)], [12, Theorem 5.1]).

If $m=-3$, then $(-\omega)-1=\omega^{2}$ in $R=\mathbb{Z}[\omega]$, where $\omega=\frac{1}{2}(-1 \pm \sqrt{-3})$ and we can choose $a=-\omega$ and $b=1$. If $m<-3$, then condition (5.2) does not hold since $R^{\times}=\{ \pm 1\}$.

Assume now that $m>0$. Then there exists a fundamental unit $\varepsilon \in R^{\times}$such that $R^{\times}=$ $\left\{ \pm \varepsilon^{n} \mid n \in \mathbb{Z}\right\}$ (see [2, Theorem 37], [12, Theorem 5.25]). Since $R\left(\varepsilon^{n}-1\right) \subseteq R(\varepsilon-1)$ when $n \neq 0$ and

$$
R\left(\varepsilon^{n}+1\right)+R\left(\varepsilon^{m}+1\right)=R\left(\varepsilon^{n}+1\right)+R\left(\varepsilon^{m}-\varepsilon^{n}\right) \subseteq R\left(\varepsilon^{n}+1\right)+R(\varepsilon-1)
$$

where $m \neq n$, we only need to consider the case when $a=-\varepsilon^{n}(n>0)$ and $b=\varepsilon$.

Suppose that $\varepsilon=\frac{1}{2}(x+y \sqrt{m})$ for some odd integers $x, y$. Then

$$
(\varepsilon+1)(\varepsilon-1-x)=-\frac{x^{2}-y^{2} m}{4}-x-1= \pm 1-x-1
$$

is an odd integer. Since $(\varepsilon+1)-(\varepsilon-1)=2$, we have $R(\varepsilon+1)+R(\varepsilon-1)=R$. For example, when $m=5$ we can take $\varepsilon=\frac{1}{2}(1+\sqrt{5})$ and hence condition (5.2) holds.

Suppose that $\varepsilon=x+y \sqrt{m}$ for some integers $x$ and $y$. Put $\varepsilon^{n}=x^{\prime}+y^{\prime} \sqrt{m}$ for some integers $x^{\prime}, y^{\prime}$. Since $x^{2}-y^{2} m= \pm 1$ and $x^{\prime 2}-y^{\prime 2} m= \pm 1$, we have $x-1 \equiv y \bmod 2$ and $x^{\prime}+1 \equiv y^{\prime} \bmod 2$. Then

$$
\varepsilon^{n}+1=x^{\prime}+1+y^{\prime} \sqrt{m}=2 \cdot \frac{x^{\prime}+1+y^{\prime} \sqrt{m}}{2}
$$

and

$$
\varepsilon-1=x-1+y \sqrt{m}=2 \cdot \frac{x-1+y \sqrt{m}}{2} .
$$

Since $R\left(\varepsilon^{n}+1\right)+R(\varepsilon-1)=2 R \neq R$ condition (5.2) does not hold. For example, when $m=37$ we can take $\varepsilon=6+\sqrt{37}$ and hence condition (5.2) does not hold. 
Therefore we have the following corollary.

COROLlaRy 5.18. Let $R$ be the ring of integers of the quadratic field $\mathbb{Q}(\sqrt{m})$. Then there exists a two-dimensional representation with Borel mold over $R$ for the free group $F_{2}$ of rank two if and only if one of the following conditions is satisfied:

(1) $m=-3$; or

(2) $m>0, m \equiv 5 \bmod 8$ and $\varepsilon=\frac{1}{2}(x+y \sqrt{m}) \in R^{\times}$for some odd integers $x$ and $y$.

\section{References}

[1] R. Fricke and F. Klein, Vorlesungen über die Theorie der Automorphen Funktionen, Vol. 1 (B. G. Teubner, Leipzig, 1897).

[2] A. Fröhlich and M. J. Taylor, Algebraic Number Theory, Cambridge Studies in Advanced Mathematics, 27 (Cambridge University Press, Cambridge, 1993).

[3] R. Hartshorne, Algebraic Geometry, Graduate Texts in Mathematics, 52 (Springer, New YorkHeidelberg, 1977).

[4] F. Hirzebruch, Topological Methods in Algebraic Geometry (Springer, New York, 1966).

[5] A. D. King, 'Moduli of representations of finite dimensional algebras', Q. J. Math. Oxf. II Ser. 45 (1994), 515-530.

[6] K. Nakamoto, 'The moduli of representations with Borel mold', arXiv:1008.0242.

[7] K. Nakamoto, 'Representation varieties and character varieties', Publ. Res. Inst. Math. Sci. 36 (2000), 159-189.

[8] K. Nakamoto and T. Torii, 'Rational homotopy type of the moduli of representations with Borel mold', Forum Math., in press; doi:10.1515/FORM.2011.071.

[9] K. Nakamoto and T. Torii, 'Topology of the moduli of representations with Borel mold', Pacific J. Math. 213 (2004), 365-387.

[10] M. S. Narasimhan and C. S. Seshadri, 'Holomorphic vector bundles on a compact Riemann surface', Math. Ann. 155 (1964), 69-80.

[11] M. S. Narasimhan and C. S. Seshadri, 'Stable and unitary vector bundles on a compact Riemann surface', Ann. of Math. (2) 82 (1965), 540-567.

[12] T. Takagi, 'Shotô Seisûron Kougi', in: Lecture on Elementary Number Theory, 2nd edn (Kyoritsu Shuppan, Tokyo, 1971), in Japanese.

KAZUNORI NAKAMOTO, Center for Life Science Research,

University of Yamanashi, Yamanashi 409-3898, Japan

e-mail: nakamoto@yamanashi.ac.jp

TAKESHI TORII, Department of Mathematics, Okayama University,

Okayama 700-8530, Japan

e-mail: torii@math.okayama-u.ac.jp 